\title{
Gradhiva
}

GRADHIV

Revue d'anthropologie et d'histoire des arts

19 | 2014

"L'Atlantique Noir » de Nancy Cunard. Negro Anthology 1931-1934

\section{Introduction « L'Atlantique noir » de Nancy \\ Cunard, Negro Anthology, 1931-1934}

\section{Sarah Frioux-Salgas}

\section{(2) OpenEdition}

\section{Journals}

Édition électronique

URL : http://journals.openedition.org/gradhiva/2771

DOI : $10.4000 /$ gradhiva.2771

ISSN : 1760-849X

Éditeur

Musée du quai Branly Jacques Chirac

Édition imprimée

Date de publication : 1 mars 2014

Pagination : 4-29

ISBN : 978-2-35744-073-9

ISSN : 0764-8928

Référence électronique

Sarah Frioux-Salgas, « Introduction « L'Atlantique noir » de Nancy Cunard, Negro Anthology, 1931-1934

», Gradhiva [En ligne], 19 | 2014, mis en ligne le, consulté le 22 septembre 2020. URL : http://

journals.openedition.org/gradhiva/2771; DOI : https://doi.org/10.4000/gradhiva.2771 
훙

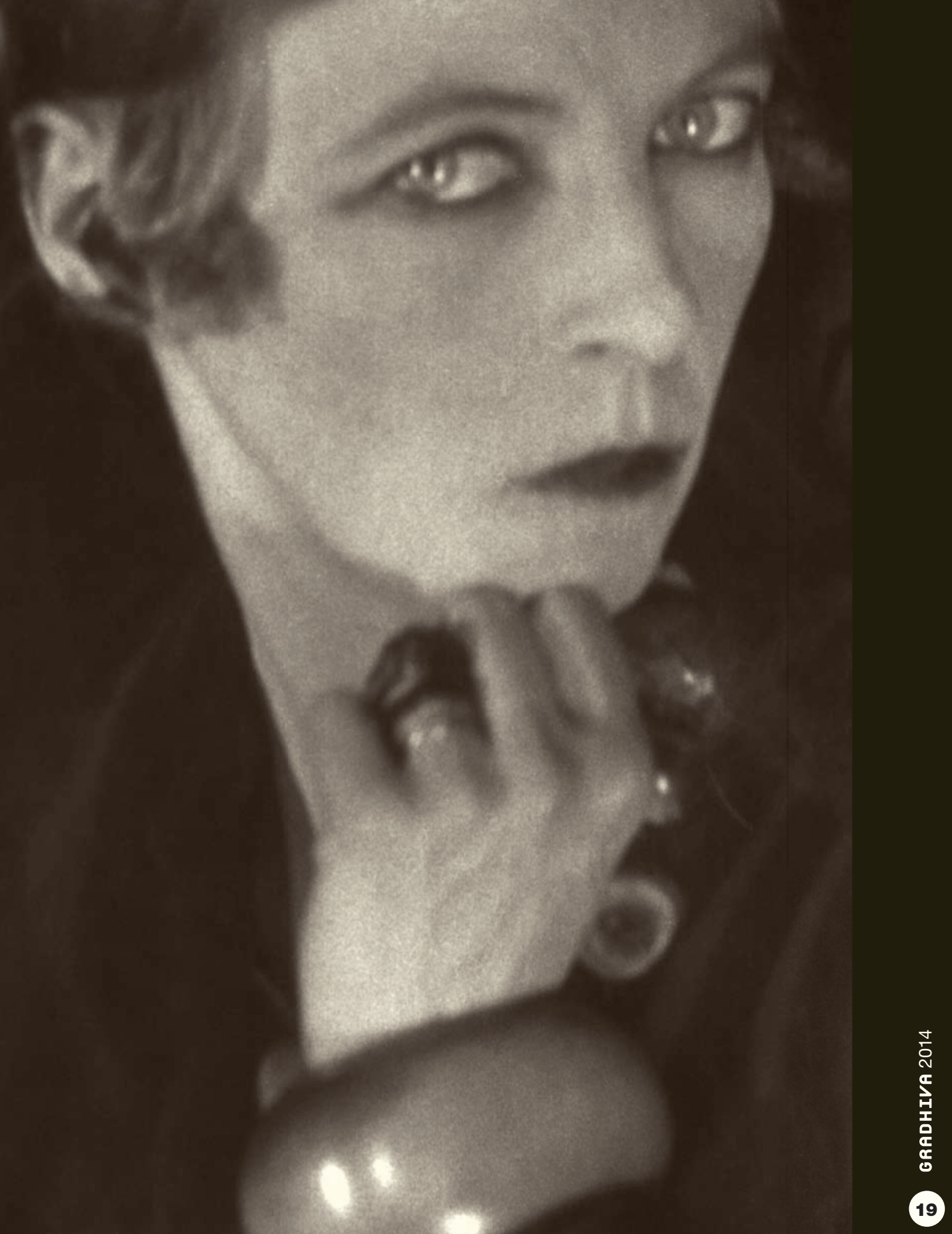




\title{
Introduction
}

\author{
«L'Atlantique noir » de Nancy Cunard, \\ Negro Anthology, 1931-1934
}

\author{
par Sarah Frioux-Salgas
}

Que vous dire de moi-même? J'aime: la paix, la campagne, l'Espagne républicaine et l'Italie antifasciste, les Noirs et leur culture africaine et afro-américaine, toute l'Amérique latine que je connais, la musique, la peinture, la poésie et le journalisme. J'ai toujours vécu en France depuis que j'en ai eu la possibilité en 1920. Je hais: le fascisme [...]. Et le snobisme et tout ce qui va avec. Deux œuvres: Negro, grande anthologie sur les Noirs; Authors Take Sides, enquête adressée aux écrivains britanniques au sujet de la guerre d'Espagne et du fascisme. Plusieurs volumes de poèmes. Nancy Cunard, Poèmes à la France. 1939-1944, Paris, Seghers, 1947, p. 7.

II y a quatre-vingts ans, le 15 février 1934, Negro Anthology de l'Anglaise Nancy Cunard ${ }^{\mathbf{1}}$ sort en mille exemplaires chez l'éditeur londonien Wishart and Company. Très illustré, ce livre de huit cent cinquante-cinq pages, dédié à l'histoire de l'Afrique, de Madagascar et des Amériques noires, réunit deux cent cinquante articles et cent cinquante-cinq auteurs ${ }^{2}$. L'aspect unique et original de cette somme tient non seulement à son concept et à sa réalisation, semblable à une grande enquête documentaire, mais également à ses auteurs. Les contributeurs sont militants, intellectuels, journalistes, artistes, poètes, universitaires, anthropologues, Africains-Américains, Antillais, Africains, Malgaches, Latino-Américains, Américains, Européens, femmes et hommes. Certains d'entre eux sont colonisés, discriminés, ségrégués.

L'ouvrage mêle culture populaire, sociologie, politique, histoire, ethnologie, histoire de l'art, et rassemble des articles, des archives, des photographies, des dessins, des portraits, des extraits de presse, des poèmes, des partitions musicales, des témoignages ou encore des statistiques.

Negro Anthology est aussi le reflet d'une histoire intellectuelle et politique de son temps. Celle-ci révèle à la fois le caractère transnational et multiforme des combats antiracistes et anticolonialistes des années 1930, et illustre la formation internationale et transculturelle que le sociologue Paul Gilroy a nommée «l'Atlantique noir ${ }^{3}$ ». Raymond Michelet, jeune collaborateur de Cunard et proche des surréalistes, témoigne: il s'agissait de «[...] montrer, démontrer que le préjugé racial ne repose sur aucune justification [...], que les Noirs avaient derrière eux [...] une longue histoire sociale et culturelle» (Ford 1968: 128). Cunard met en œuvre sa démonstration en proposant diverses approches, différents points de vue, et propose ainsi «d'effectuer un voyage intellectuel à travers l'Atlantique noir » (Gilroy 2003: 19). Nous nous attacherons à montrer, dans cet article et ce numéro, comment elle construit cet ouvrage politique d'une grande modernité formelle et théorique grâce à ses réseaux, ses amitiés, ses activités intellectuelles et éditoriales, ses convictions radicales et son engagement total.

\begin{abstract}
1. Nancy Cunard
(10 mars 1896-15 mars 1965). Plusieurs biographies lui sont consacrées par Hugh Ford (1968), Anne Chisholm (1980) et plus récemment François Buot (2008). L'ouvrage de Ford est particulièrement intéressant car il rassemble de nombreux témoignages très éclairants sur les multiples vies de Cunard.
\end{abstract}

\section{Une petite carte publicitaire pour l'anthologie indique qu'il y a 385 illustrations et que son prix est de 2,2 livres (équivalant aujourd'hui à environ 80 euros). Par ailleurs, Ford indique que Cunard investit personnellement 1500 livres (environ 60000 euros aujourd'hui) pour produire l'anthologie.}

\author{
3. Paul Gilroy explique \\ la formation politique \\ et culturelle de ce qu'il \\ appelle Black Atlantic \\ de la façon suivante: \\ «peut être définie à travers \\ le désir de transcender \\ à la fois les structures de la \\ nation et les contraintes de \\ l'ethnicité et du particularisme \\ national " (Gilroy 2003: \\ 38). Il estime que cette \\ formation est profondément \\ «interculturelle» et est \\ alimentée par des \\ «cultures diasporiques \\ rhisomorphiques". \\ Pour une étude critique \\ des travaux de Gilroy, \\ voir Chivallon 2004.
}




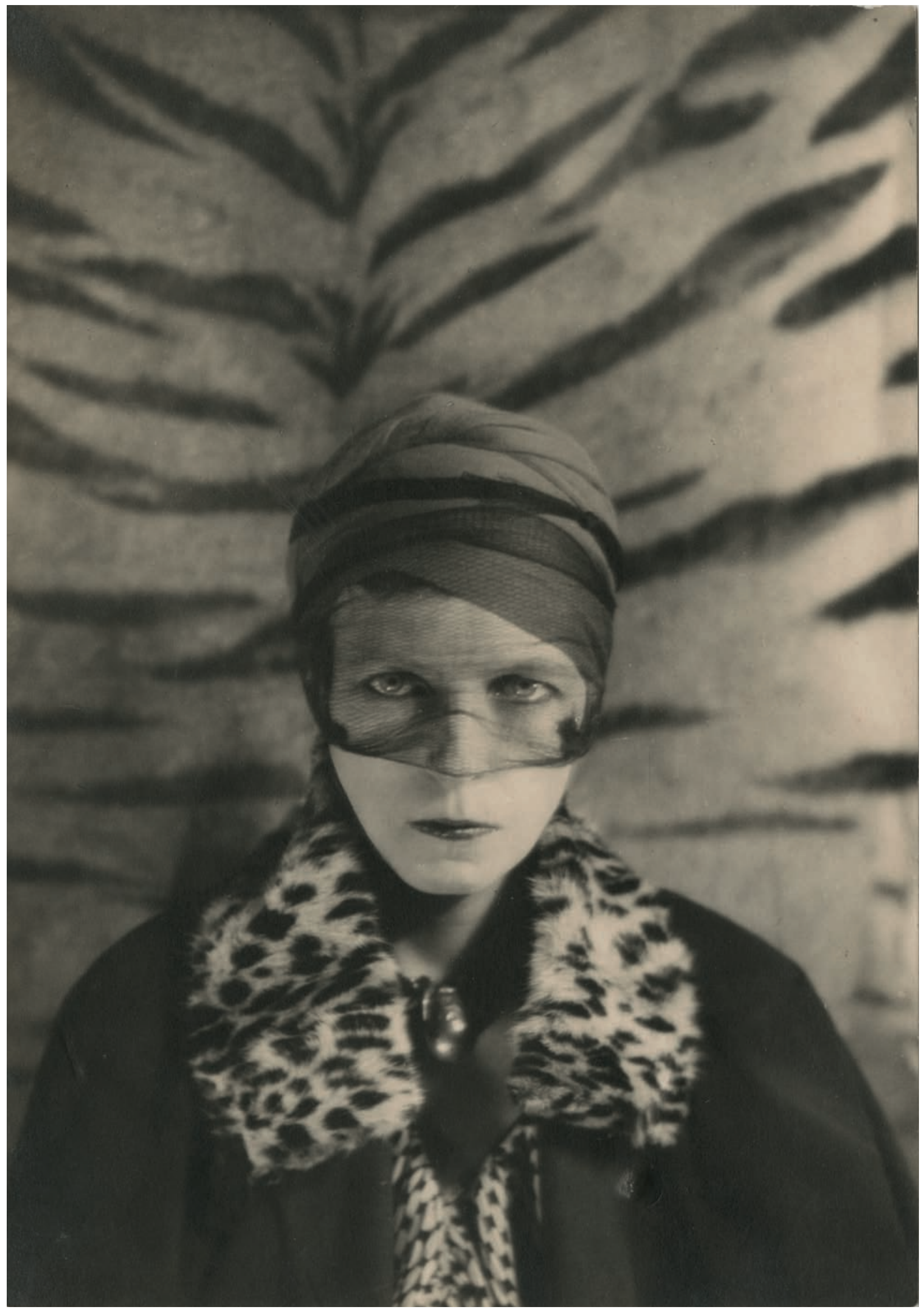




\section{Nancy Cunard, une femme-époque}

Cunard, symbole de l'avant-garde anglo-saxonne et française des années 1920, est née en Angleterre en 1896. Sa mère, Maud Alice Burke, est américaine et son père, Bache Cunard, anglais, héritier de l'entreprise maritime Cunard Line. Elle passe son enfance au château de Neville Holt, dans le centre de l'Angleterre, élevée par des gouvernantes au rythme des fêtes organisées par sa mère, Lady Cunard. Adolescente, elle voyage, étudie dans une école prestigieuse de Londres, en France et en Allemagne, et suit sa mère dans ses activités mondaines. C'est à la veille de la première guerre mondiale, à ses 18 ans, qu'elle entame sa vie de jeune fille libre, bohème et provocante qui cherche à s'affranchir des règles de l'Angleterre victorienne. Son amie Iris Tree ${ }^{4}$ témoigne:

[...] À ce moment-là, nous étions des bandits, n'hésitant pas à nous maquiller avec de la craie blanche sur le visage et du rouge à lèvres écarlate, fumant des cigarettes parmi des fêtards choisis par nous-mêmes [...] Nous étions de vrais caméléons. Nous passions de Meredith à Proust et à Dostoïevski, goûtions à l'absinthe avec Baudelaire et Oscar Wilde, [...] nous nous laissions assombrir par le pessimisme nihiliste, [...] inspirées par le jeune Rupert Brooke, T.S. Eliot, Yeats, D.H. Laurence, secouées par Blast de Wyndham Lewis, [...] la «signifiant form ", [...], les sculptures d'Epstein, la musique de Stravinsky, les premiers ballets russes et le jazz américain » (Ford 1968: 19, notre traduction)

Ce témoignage ancre clairement Cunard dans la contre-culture anglaise du début du $x x^{e}$ siècle. Wyndham Lewis, T.S. Eliot, Jacob Epstein mais aussi Ezra Pound, qui n'apparaît pourtant pas dans cette liste, mais qui fut un temps proche de Cunard, sont imagistes ${ }^{5}$ ou vorticistes $^{6}$, deux mouvements artistiques et littéraires qui symbolisent la modernité et la radicalité anglo-saxonne avant la première guerre mondiale.

Cunard veut aussi être poète. Ses premiers poèmes sont publiés en 1916 dans le premier numéro de l'anthologie Wheels, titre d'un de ses poèmes, éditée par les frères Sitwell ${ }^{7}$ et consacrée à la «nouvelle poésie». Elle est également l'auteur de quatre recueils de poésies: Outlaws (1921), Sublunary (1923), Parallax (1925) et Poems (Two) (1930). À Londres, elle fréquente régulièrement le “Bloomsbury group 8 ", qui regroupe des théoriciens, des écrivains et des peintres dont les époux Leonard et Virginia Woolf, John Maynard Keynes ou encore Clive Bell et Roger Fry, qui développent notamment le concept de la «signifiant form ${ }^{9}$ " que Tree évoque dans ses souvenirs. En 1917, les époux Woolf fondent Hogarth Press, maison d'édition majeure dans l'histoire politique et littéraire britannique ${ }^{\mathbf{1 0}}$, et éditent en 1925 Parallax. À cette époque, Cunard est aussi le symbole d'une certaine élite anglaise meurtrie par la première guerre mondiale, qui se bat contre l'Angleterre traditionnelle et défend une nouvelle liberté des mœurs. Iris March, héroïne du roman de Michael Arlen devenu culte, Le Chapeau vert (1924), qui décrit ce milieu, est officiellement inspirée par Cunard, qui fut un temps la compagne de l'auteur.

Dans les années 1920, Cunard voyage beaucoup en Europe, et vit entre Londres et Paris. La capitale française accueille alors de nombreux
4. Iris Tree (1897-1968): poète et actrice liée à l'avantgarde anglo-saxonne des années 1920. Elle fut mariée au photographe Curtis Moffat entre 1916 et 1932.

5. Mouvement littéraire qui devait aboutir au renouvellement radical de la poésie anglaise. Ce terme fut inventé en 1913 par l'écrivain américain Ezra Pound qui, à cette époque, vivait en Angleterre. Les poètes de ce mouvement étaient essentiellement anti-victoriens et antiromantiques.

6. La revue Blast citée par Tree est fondée avec l'aide de Pound par Wyndham Lewis. Il n'en existe que deux numéros, publiés en 1914 et 1915. L'objectif de Blast était de promouvoir un nouveau mouvement dans la littérature et les arts visuels, baptisé « vorticisme " par Pound et Lewis.

Les influences du vorticisme sont, en littérature, l'imagisme et, en arts plastiques, le cubisme et le futurisme. Par ailleurs, Cunard et Lewis eurent en 1922 une aventure au cours de laquelle celui-ci réalisa son portrait, publié dans la revue Sketch et qui illustre aussi la page de garde de Sublunary, ouvrage de poésie de Cunard publié en 1923.

\section{ci-contre}

fig. 1

Barbara Ker-Seymer, Nancy Cunard, 1930. Harry Ransom Center. The University of Texas, Austin. Archives Nancy Cunard: 957:0038:031 (C) The Estate of Barbara Ker-Seymer.
7. Les frères Sitwell et Edith Sitwell appartiennent à cette nouvelle génération Iondonienne qui souhaitait défendre une poésie contemporaine et moderne. Ils publieront eux aussi leurs poèmes dans Wheels (1916-1921).

8. Ce groupe commence à se réunir en 1904 tous les jeudis dans le quartier de Bloosmbury où s'étaient installés les époux Woolf.

9. Clive Bell expose cette théorie dans Art, publié en 1913. La "forme signifiante», théorie qui prône entre autres l'importance de la forme au détriment des contenus, s'inscrit dans les mouvements d'idées modernistes de l'histoire intellectuelle anglaise du début du $x x^{e}$ siècle.

10. Ils publient les auteurs modernistes anglo-saxons de leur temps et traduisent de nombreux auteurs étrangers, notamment Sigmund Freud, Italo Svevo, Rainer Maria Rilke, Maxime Gorki, Léon Tolstoï. 

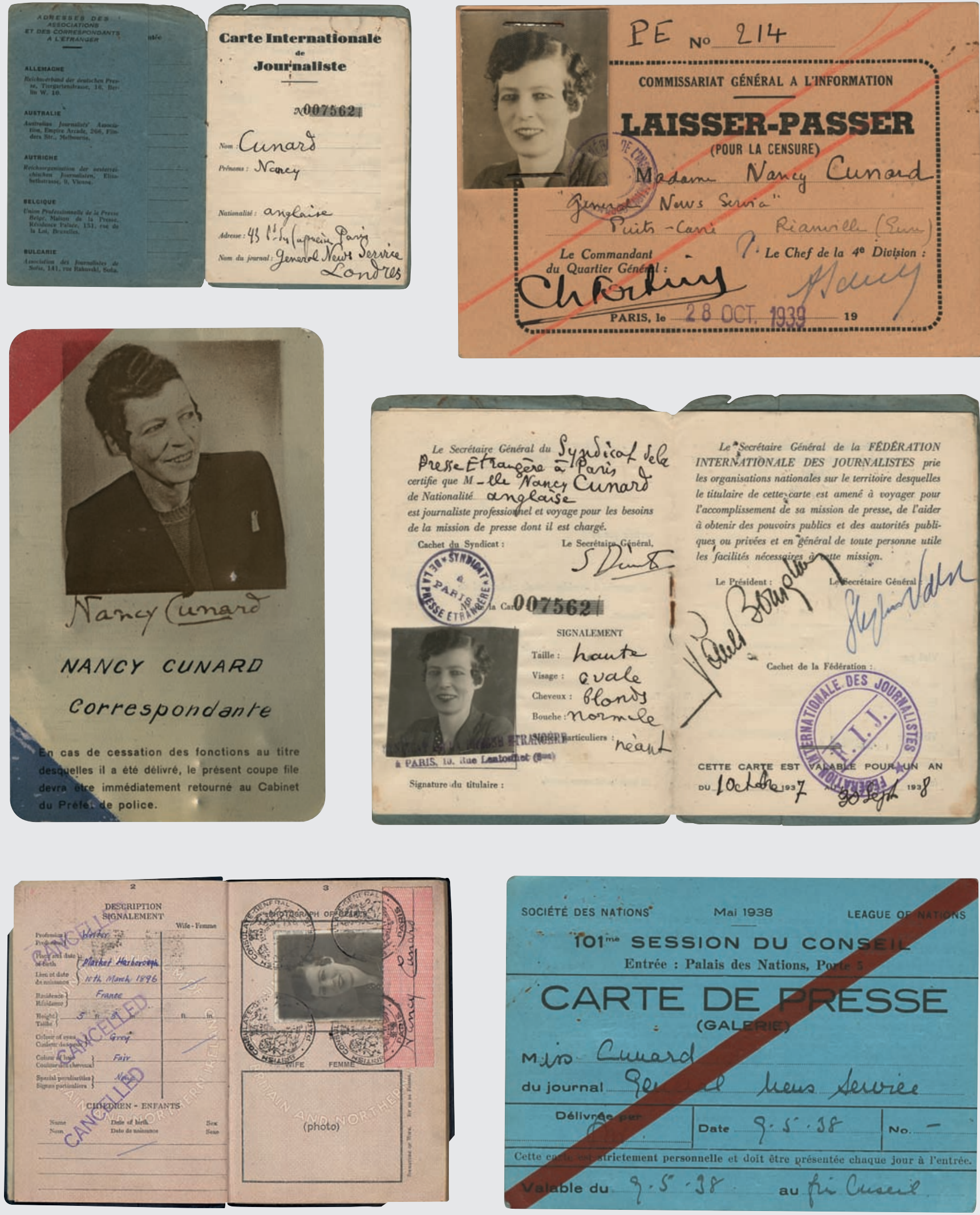

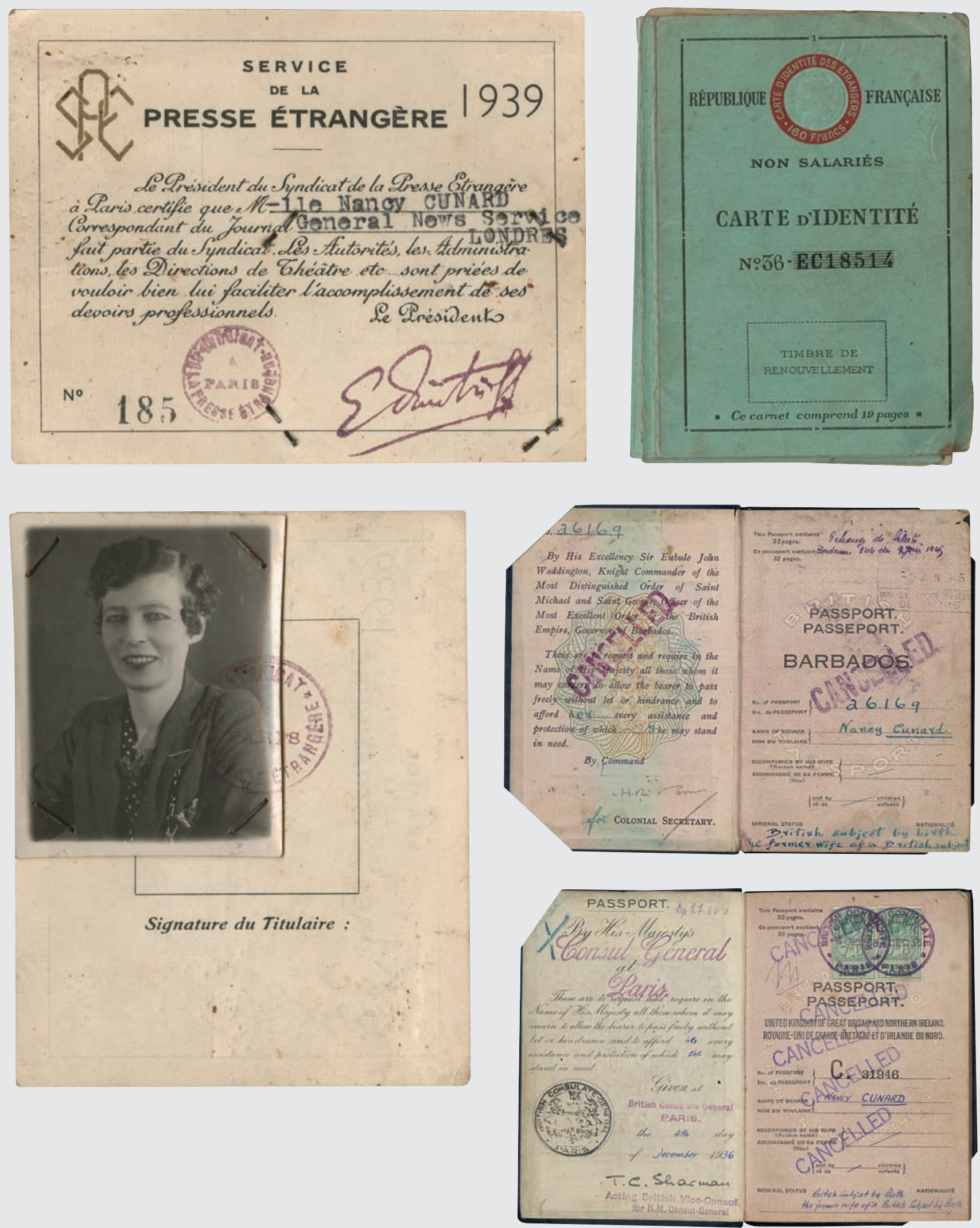
11. Lionel Follet cite Adrienne Monnier dans la préface de l'édition renouvelée et augmentée de La Défense de l'infini de Louis Aragon (Aragon 2002).

12. Voir le portrait de Janet Flaner par Berenice Abbott, 1927 (Washington D.C., Library of Congress), où elle porte le manteau du père de Cunard. Cette photographie fut exposée en 1928 à la Comédie des Champs-Élysées au premier salon indépendant de la photographie. En 1924, le peintre américain Eugene MacCown réalise un portrait de Cunard (conservé aux archives d'Austin) et Man Ray la photographie, en compagnie de Tristan Tzara, au bal du comte de Beaumont.

13. À qui Cunard commande en 1924 un chapeau et manteau. En 1925, elle pose ainsi vêtue pour le photographe Curtis Moffat. Photographie conservée à Londres, au Victoria and Albert Museum (E.1563-2007).

14. Un tapuscrit de cette pièce avec une dédicace de Tzara se trouve au Harry Ransom Center à l'université du Texas à Austin, où sont conservées les archives de Cunard. Man Ray immortalise leur amitié dans une photographie de 1925.

15. Jean-Michel Frank (1895-1941) était un ami de lycée de René Crevel.

16. Paris, archives Jacques Doucet, fonds Marcel Jouhandeau.

\section{double page \\ précédente}

fig. 2

Ensemble de pièces d'identité et de cartes de presse de Nancy Cunard. Harry Ransom Center. The University of Texas, Austin. Archives Nancy Cunard: Box 21/folder 8 .
Anglo-Saxons, journalistes, écrivains, artistes, photographes, éditeurs, mécènes, poètes, qui seront tous, à un moment ou à un autre, de vrais Parisiens tel que l'entend Valéry Larbaud: "On est parisien dans la mesure où on contribue à l'activité matérielle et à la puissance spirituelle de Paris ${ }^{11}$." En effet, à cette époque, Paris héberge de nombreuses maisons d'édition et revues anglaises et américaines: Contact Edition, Three Mountains Press, The Little Review, This Quarter, Transatlantic Review, Transition... Shakespeare and Company, première librairie de prêt anglophone, fondée en 1919 par l'Américaine Sylvia Beach dans le quartier de l'Odéon, diffuse ces nombreuses publications aux côtés de celles éditées en Angleterre ou aux États-Unis (The Dial, The Egoist, The News Masses...). La librairie édite aussi Ulysses de Joyce en 1922.

Cunard est une de ces femmes anglo-saxonnes qui ont quitté leur pays trop puritain et se sont installées à Paris pour vivre pleinement leur sexualité ou leurs activités intellectuelles et créatrices (Chadwick et Latimer 2003). Les couples que forment Gertrude Stein et Alice B. Toklas d'une part, Natalie Barney et Romaine Brooks d'autre part, en sont les exemples les plus célèbres. Cunard, quant à elle, forme un trio original et extravagant avec le couple de journalistes américaines Janet Flaner et Solita Solano (Broderick 1977; Ford 1968), rencontrées en 1923. Plusieurs peintres et photographes les ont d'ailleurs immortalisées en chapeaux haut-de-forme et vestes de cavalière ${ }^{\mathbf{1 2}}$. Symbole de la «mode garçonne» des années 1920, cette tenue, inspirée des dandys du xix ${ }^{e}$ siècle, représentait, pour ces femmes émancipées, la modernité et la remise en question de la masculinité (Chadwick et Latimer 2003). Malgré la réalité plus complexe du statut des femmes en métropole (Boittin 2010; Latimer 2005), la capitale française était, à cette époque, un espace de liberté pour les femmes étrangères.

Cunard est aussi une femme riche et mondaine habillée par Paul Poiret, Elsa Schiaparelli, Coco Chanel ou Sonia Delaunay ${ }^{13}$. Elle participe aux nombreuses fêtes parisiennes qui rassemblent artistes, écrivains et poètes d'avant-garde ainsi que les élites qui les soutiennent. À partir de 1923, elle devient très proche de Tristan Tzara puis de René Crevel. L'année suivante, Tzara lui dédie sa pièce Mouchoir de nuages ${ }^{14}$. À partir de 1924, son appartement de l'île Saint-Louis, rue Le Regrattier, aménagé par le décorateur Jean-Michel Frank ${ }^{\mathbf{1 5}}$, devient un lieu de rencontre entre les intellectuels anglo-saxons et l'avant-garde littéraire et artistique parisienne. Elle joue alors, de manières assez variées, le rôle d'intermédiaire, de passeuse ou de traductrice entre ces milieux. En 1924, elle traduit en français, pour Tzara, la pièce Faust de Christopher Marlowe, puis pour Crevel la version anglaise d'un classique japonais, Le Dit du Genji (Buot 2008: 126). En 1926, elle propose à l'éditeur l'anglais John Rodker (éditions Ovid) de traduire en anglais le premier roman de l'écrivain Marcel Jouhandeau, Mademoiselle Zéline ${ }^{16}$ (1924). Elle tient aussi une chronique régulière, "Paris today as I see it", dans la version anglaise du magazine Vogue. En mai 1926, elle y décrit l'exposition Tableaux de Man Ray et objets des îles présentée à la Galerie surréaliste (Grossman 2009). Cette même année, Man Ray réalise une série de portraits d'elle, dont le plus célèbre en habit léopard et cheveux courts avec au premier plan ses bras recouverts de bracelets africains. Le 5 octobre 1927, le Vogue anglais publie cette photographie légendée 
«London fashion» et accompagnée d'un petit texte la présentant comme une jeune poétesse vivant à Paris (ibid.: 136). Cette image fut aussi reproduite en 1929 dans deux autres revues, une belge, Variétés, Revue mensuelle illustrée de l'esprit contemporain, et une américaine, The Little Review (ibid.: 147). Ce portrait mythique est en quelque sorte une synthèse de l'histoire artistique et culturelle des années 1920, largement étudiée ${ }^{\mathbf{1 7}}$, que l'on associe souvent à la femme émancipée, au goût pour les arts non occidentaux et au primitivisme.

Avant Man Ray, des peintres comme Lewis, Eugene MacCown, John Banting ou Oskar Kokoschka avaient déjà réalisé des portraits de Cunard ${ }^{18}$, mais à partir de $1926^{19}$ elle devient le modèle d'autres grands photographes anglo-saxons. Curtis Moffat (ancien mari de son amie Iris Tree), Cecil Beaton 20 et Barbara Key-Seymer, tous sensibilisés à l'esthétique surréaliste ${ }^{\mathbf{2 1}}$, réalisent de magnifiques clichés qui confirment son statut d'icône de l'entre-deux-guerres.

Sa liaison avec l'écrivain Louis Aragon, qui dure officiellement de 1926 à 1928, condense aussi l'histoire intellectuelle des années 1920. L'année où ils se rencontrent, ce dernier publie intégralement son roman surréaliste Le Paysan de Paris ${ }^{\mathbf{2 2}}$ et s'implique de plus en plus dans la rédaction de La Défense de l'infini commencée vers 1923. Au côté de Cunard, écrit-il à Jacques Doucet, «je suis continûment heureux pour la première fois de ma vie» (préface de Follet in Aragon 2002: XXVII). Un passage de La Défense de l'infini semble évoquer sa compagne. Armand, l'un des personnages principaux du roman, décrit la femme qu'il aime: «une fille grande ouverte à l'avenir [...] félonne et féline [...]. Délicieux tombeaux; grande fille du temps $[. . .]^{23}$ ". Dix-neuf chapitres de ce roman, qu'Aragon a essayé de faire disparaître de sa bibliographie pendant quarante ans, ont été retrouvés dans les archives de Cunard ${ }^{24}$ (ibid.). Aragon a plusieurs fois évoqué l'autodafé (jamais prouvé) d'une partie du manuscrit lors d'un séjour commun à Madrid en 1927. Cette même année, Cunard est aussi à ses côtés, en Normandie, lorsqu'il amorce sa rupture formelle avec le surréalisme en écrivant Traité de style (Paris, Gallimard, 1928), à quelques kilomètres du lieu de villégiature de leur ami André Breton, qui entame alors Nadja. L'année suivante, les activités de «passeuse littéraire» de Cunard continuent avec la traduction (ou son financement) du chapitre d'ouverture de Nadja, en mars 1928, pour la revue américaine Transition d'Eugene Jolas ${ }^{25}$.

Sensibilisée aux arts africains et océaniens, au début des années 1920, par Moffat ${ }^{26}$ (Cunard 1969: 80), c'est avec Aragon que Cunard entame sa collection d'art non occidental (Aragon 2011; Grossman 2009). Comparativement aux autres collections importantes de l'époque, la sienne ne rassemble pas d'œuvres exceptionnelles. Elle est surtout célèbre pour sa spécificité: les ivoires, et plus particulièrement les bracelets; Cunard en possède environ cinq cents. En 1955, elle entreprend la réalisation d'un ouvrage sur les ivoires africains qui ne voit pas le jour, malgré de nombreuses informations collectées. Si, en 1930, Man Ray en photographie certains, c'est l'artiste belge Raoul Ubac qu'elle choisit, en 1931, pour «mettre en scène" une partie de ses pièces ${ }^{27}$. Pendant la seconde guerre mondiale, la majorité de sa collection disparaît. Une partie aurait été volée, l'autre
17. Jean Jamin, James Clifford, Denis Hollier, Jean-Louis Paudrat, John Ruskin et plus récemment Yaëlle Biro, Benoît de l'Estoile ou Sophie Leclercq. Voir aussi les travaux de Wendy A. Grossman.

18. En 1925, Constantin Brancusi sculpte un portrait d'elle qu'il intitule Une jeune fille sophistiquée, dont une version en plâtre est aujourd'hui conservée au centre Georges-Pompidou. Dans l'ouvrage de Hugh Ford (1968), Marcel Duchamp raconte que, pour la réalisation de cette sculpture (donnée à sa femme Teeny par Brancusi dans les années 1950), Cunard n'avait pas posé mais que le sculpteur s'était inspiré de son personnage sans qu'elle le sache. Elle l'apprit plus tard.

19. Une liste réalisée par Cunard des dessins et peintures (sans les photographies) la représentant est consignée dans un cahier conservé dans ses archives. Elle liste 36 portraits d'elle réalisés entre 1905 et 1957 (archives Nancy Cunard).

20. Cecil Beaton (1904-1980) est un des plus célèbres photographes de portraits anglais. Sa première grande exposition a lieu en 1927. Avec le soutien de la famille Sitwell, il devient un collaborateur de la version anglaise de Vogue pendant cinquante ans. En 1930, il sélectionne une de ses photographies de Cunard pour son livre The Book of Beauty.
21. John Banting, artiste surréaliste anglais, décore le studio de Barbara Key-Seymer pour la séance de prise de vues de Cunard en 1930.

22. Plusieurs passages avaient déjà été publiés dans La Revue européenne.

23. Ce passage consacré à Cunard et Aragon est en grande partie rédigé à partir de la passionnante édition renouvelée et augmentée par Lionel Follet de La Défense de l'infini (Aragon 2002).

24. Ces chapitres ont été retrouvés en 1991 par Lionel Follet.

25. Le manuscrit de cette traduction anglaise appartenait aux archives André Breton. Voir également l'article de Thomas Hunkeler "Beckett face au surréalisme" dans Samuel Beckett Today/ Aujourd'hui, vol. XVII, 2006.

26. Moffat était lui aussi collectionneur. Wendy A Grossman signale un article d'avril 1935 sur la collection de Moffat dans la revue anglaise The Connoisseur ( $\left.n^{\circ} 104\right)$ : "Primitive art of Africa and the Polynesian islands in the collection of Mr Curtis Moffat ».

27. Le musée du quai Branly conserve dans ses collections un certain nombre de ces photographies données en 1932 par Cunard. Voir dans ce numéro l'annexe «Photographies de la collection personnelle de Nancy Cunard par Raoul Ubac, aujourd'hui au musée du quai Branly». 


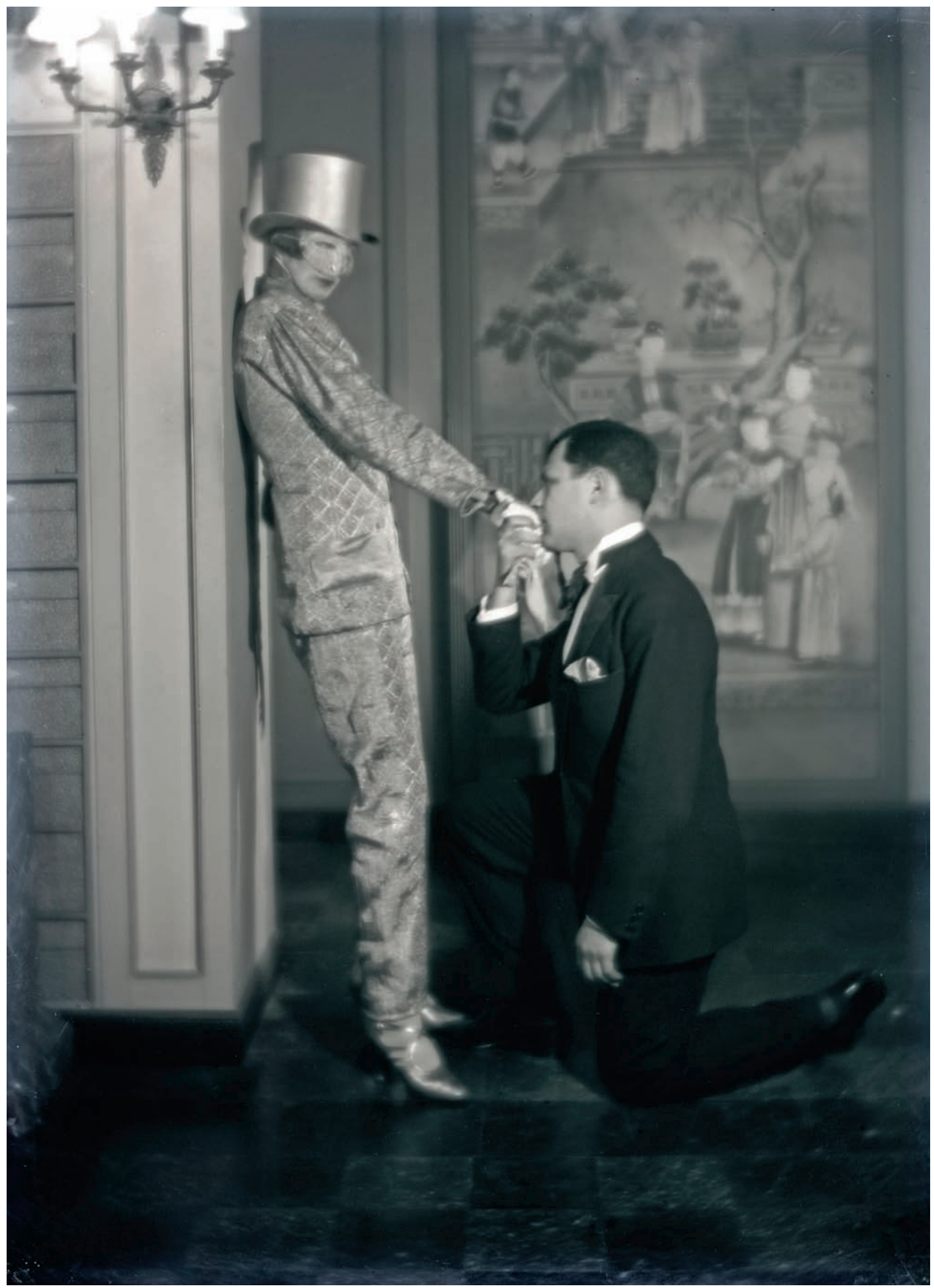


saccagée par des soldats nazis et par les habitants du village normand où elle vivait 28 .

C'est encore avec Aragon qu'elle fonde, en 1928, sa maison d'édition, Hours Press ${ }^{29}$, qui devait défendre «l'innovation et une nouvelle vision des choses »(Cunard 1969) et publier de la poésie expérimentale. Aragon raconte:

Nane avait acheté une petite maison avec un jardin, quelque part, au-delà de Vernon, c'est-à-dire un peu au nord-ouest de Vernon, me semble-t-il. Le jeu avait commencé d'installer ici les retours des voyages. Nous faisions presque tout de nos mains, les peintures, aménager une sorte de hangar, pour un projet assez fou, une imprimerie, la presse à bras... un métier à apprendre... composer à la main... est-ce qu'on sait encore ce que c'est aujourd'hui? J'y avais mis toute ma folie. [...] Mon projet était d'imprimer une traduction de Lewis Caroll, un texte en France inconnu, La Chasse au Snark. Tout devait y être de ma main, y compris les caractères de la couverture, inventés par moi. Près d'un an y passa. La maison était devenue la folie de Nancy. Enfin, je ne vais pas raconter ça... (Aragon 1974: 34)

Elle découvre le métier d'éditeur-imprimeur avec Aragon, mais c'est surtout avec son nouveau compagnon Henry Crowder, pianiste africainaméricain rencontré à Venise, l'été 1928, qu'elle le pratique pendant quatre ans (Crowder et Speck 1987; Barnett 2007). Elle édite vingt-trois ouvrages en anglais et en tirages limités, dont le premier texte publié de Samuel Beckett, Whoroscope ${ }^{30}$, et un des volumes des Cantos de Pound. L'unique ouvrage en français est la traduction de La Chasse au Snark. Les auteurs sont déjà connus, des proches, des connaissances, tous anglo-saxons mis à part Aragon et le Chilien Alvaro Guevara. Hours Press fait partie des maisons d'édition anglo-saxonnes et françaises qui commencent, dans la première moitié $d u x x^{e}$ siècle, à publier des ouvrages limités réalisés dans des conditions artisanales, souvent en collaboration avec des artistes et destinés essentiellement à des bibliophiles (Moeglin-Delcroix 1997). Cunard propose aux auteurs de réaliser leur propre couverture ou fait appel à des artistes contemporains. Le Néo-Zélandais Len Lye ${ }^{\mathbf{3 1}}$, l'Anglais John Banting, le Français Yves Tanguy ou encore l'Américain Man Ray ${ }^{22}$ sont sollicités.

Elle utilise également sa maison d'édition pour participer aux activités de ses amis surréalistes. En 1930, elle imprime, rue Guénégaud, l'un des programmes du film L'Âge d'or de Luis Buñuel ${ }^{33}$. Elle s'implique également dans ce qu'on appelle «l'affaire de L'Âge d'or ${ }^{\mathbf{3 4}}$ » en organisant le 2 janvier 1931 une projection du film au Gaumont Theatre de Londres, après la décision de la commission de censure française. À cette occasion, elle publie une version anglaise du tract édité par les surréalistes sur les péripéties du film $^{35}$. Le 16 décembre 1930, l'exploitant de L'Âge d'or raconte l'organisation de la projection anglaise au financeur du film, Charles de Noailles:

J'ai loué une copie pour une séance privée (c'est-à-dire dans une salle louée par un groupe mais pas au grand public) en Angleterre au prix de quelque 7500 francs, je dis quelque car c'est en livres, ce qui
28. Cunard quitte la France pendant la guerre passe quelques mois en Amérique du Sud (entre autres au Chili chez Pablo Neruda), puis vit à Londres. Les documents relatifs à la disparition et au saccage de sa collection d'objets mais aussi de nombreux ouvrages et de certains tableaux à La ChapelleRéanville sont conservés dans ses archives. Ce sont selon toute vraisemblance les habitants du village puis les nazis qui pillèrent la maison, mais plusieurs documents évoquent également la venue, peu de temps après le départ de Cunard, d'une voiture noire qui serait repartie avec de gros sacs remplis d'objets. Après cette expérience traumatisante, elle vend sa maison et s'installe dans le Lot, à Lamothe-Fénelon, région qu'elle avait beaucoup fréquentée avant la guerre.

29. Après avoir installé son imprimerie dans sa maison de La ChapelleRéanville, elle décide en 1929 de la transférer à Paris, au 15, rue Guénégaud, où elle embauche Georges Sadoul comme secrétaire. Dans une annonce de 1930, elle écrit: "Les éditions Hours Press s'intéressent aussi à l'ethnographie - à l'art africain, océanien et des deux Amériques - et auront toujours quelques spécimens en exposition, ainsi que certains tableaux français modernes, quelques livres anglais et américains, et des publications surréalistes. »

\section{ci-contre}

fig. 3

Man Ray, Tristan Tzara et Nancy Cunard au bal du comte de Beaumont, 1924 (C) Man Ray Trust - Adagp, Paris 2014 @ Centre Pompidou, MNAM-CCl, Dist. RMN-Grand Palais / image Centre Pompidou, MNAM-CCI.
30. Elle publie le premier ouvrage de Samuel Beckett dans le cadre d'un concours de poésie sur le thème du temps, qu'elle lance en 1930 avec l'écrivain anglais Richard Aldington.

31. Len Lye (1901-1980) est un des pères du cinéma expérimental.

32. II réalise notamment un photomontage pour la couverture du livre musical Henry Music, dans lequel Henry Crowder met en musique des poèmes d'Aldington, Harold Acton, Beckett, Walter Lowenfels et Cunard. À ce sujet, voir Barnett 2007.

33. Ce document est conservé au centre Georges-Pompidou, bibliothèque Kandinsky, fonds Charles et Marie-Laure de Noailles.

34. Le 3 décembre 1930, au cinéma le Studio 28 , la Ligue des patriotes et la Ligue anti-juive troublent la projection et saccagent le hall du cinéma dans lequel étaient exposés des tableaux d'artistes surréalistes. Après ces événements, une deuxième commission d'examen censure le film le 11 décembre 1930. À ce sujet, voir le numéro spécial des Cahiers du MNAM de 1993 consacré au film.

35. Centre Georges Pompidou, bibliothèque Kandinsky, fonds Charles et Marie-Laure de Noailles. 
36. Centre GeorgesPompidou, bibliothèque Kandinsky, fonds Charles et Marie-Laure de Noailles.

37. Studies in Tasawwut (1923) de l'Indien Khan Khaja, The Anatomy of African Misery (1927) de Lord Olivier, Poems and Plays (1927) d'Harindranath Chattopadhyaya, Imperialism and Civilization (1928) de Leonard Woolf,

The Case for West Indian Self-Government (1933) du Trinidadais C.L.R James, An African Speaks for His People (1934) du Kényan Mockerie Parmenas Githendu, Three Guineas (1938) de Virginia Woolf.

\section{ci-contre}

fig. 4

Mon camarade: l'illustré de la jeunesse, $n^{\circ} 10$, mai 1934 Bibliothèque nationale de France, Paris, D.R. correspond à peu près à cela. C'est organisé par une personne qui a appartenu au groupe surréaliste et qui en réalité y appartient encore et messieurs Breton, Dali, etc. sont très contents que le film passe de cette manière en Angleterre et dans ce milieu.

Le 8 mars 1931, Cunard résume à Noailles la séance: «Je voulais vous envoyer ceci depuis le moment que ç'a eu lieu - mais pas d'adresse. Très bien passé en Angleterre - autrement reçu qu'à Paris [... $]^{36}$.»

En 1931, elle décide aussi de mettre un terme aux éditions Hours Press, pour des raisons financières et administratives, mais surtout pour se consacrer entièrement à la préparation de son anthologie. Dans un témoignage de 1968, Georges Sadoul résume clairement l'état d'esprit de Cunard au début des années 1930:

[...] à cette époque de romantisme révolutionnaire, où la menace d'un coup d'État fasciste n'était pas imaginaire en France, Cunard pensait aussi qu'un jour sa Press de Réanville pourrait servir à des publications illégales antifascistes. Elle était prête à se mettre à la disposition d'organisations clandestines. Cette déclaration d'intention me surprit, venant d'une dame anglaise si noble et si riche. J'en attribuais le mérite au seul Aragon. J'avais tort. La suite de la vie de Nancy me le prouva. (Ford 1968: 146, notre traduction)

\section{Negro Anthology: une riposte}

Lorsqu'elle décide de réaliser Negro Anthology en 1931, Cunard s'est déjà confrontée aux questions impériales, coloniales et raciales dans sa vie intellectuelle et personnelle. À Londres, les éditeurs de son recueil Parallax, Leonard et Virginia Woolf (Hogarth Press), publient des auteurs issus de l'Empire anglais mais aussi des textes dénonçant la situation impériale $^{37}$ depuis le début des années 1920. Par ailleurs, Cunard appartient, comme nous l'avons vu précédemment, au courant moderniste du début du $x x^{e}$ siècle qu'Edward Saïd analyse ainsi dans Culture et Impérialisme:

Ce que je voudrais suggérer, c'est que de nombreux traits cruciaux du modernisme, que nous expliquons d'ordinaire par des dynamiques purement internes à la culture et à la société occidentales, intègrent une réaction à des pressions extérieures (venue de l'imperium) sur cette culture. (Saïd 2000: 270-271)

En effet, les biographies et les pratiques littéraires de certains auteurs que Cunard convoque pour son anthologie illustrent clairement les propos de Saïd. La poétesse et journaliste anglaise Beatrice Hasting, auteur d'un texte sur l'Afrique du Sud, passa une partie de sa jeunesse dans ce pays. Pound, lui aussi contributeur, s'inspira des travaux de l'orientaliste américain Ernest Fenelossa, consacrés aux idéogrammes chinois, pour composer certains de ses Cantos. L'écrivain anglais Norman Douglas, dont des photographies de Massaï du Kenya sont reproduites, séjourna en Afrique de l'Est dans les années 1920. L'analyse de Saïd semble aussi tout à fait pertinente à propos de l'engagement anticolonialiste des surréalistes (Leclercq 2010), très présents dans l'anthologie. Cunard rassemble 


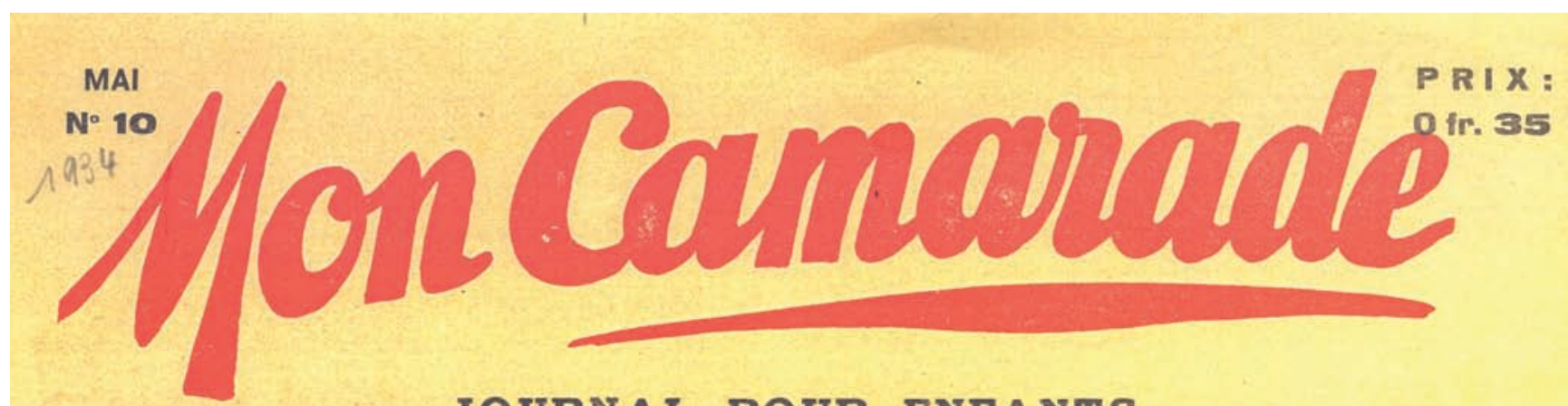

\section{JOURNAL POUR ENFANTS}

EDITE PAR LA FEOERATION DES ENFANTS OUVRIERS ET PAYSANS, 8, AV. MATHURIN-MOREAU, PARIS 19. AVEC LA COLLABORATION DE L'ASSOCIATION DES ECRIVAINS ET ARTISTES REVOLUTIONNAIRES (AEAR) M EN S U E L. Abonnement: France - 6 Nos: 2 frs.; 12 Nes: 4 frs. Etranger - 6 Nos: 4 frs.; 12 Nos: 8 frs. Envoyer l'argent à l'adrnase suivante : Chèque Postal : Paris 942-01. M. EILFA, 2, rue Robineau - (20.)

\section{LES AVENTURES DE PIPE FLUTE ET POMME ENFANTS DE PROLETAIRES}

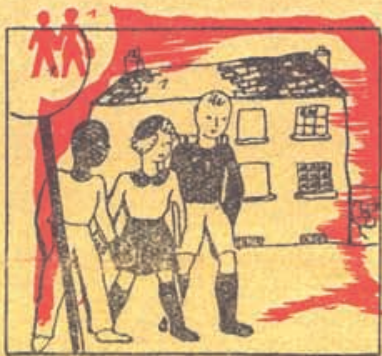

1. Davant Yésole Pipe attend to sortie de Flute et Pomme
peur rotourner ensemble chez eux.

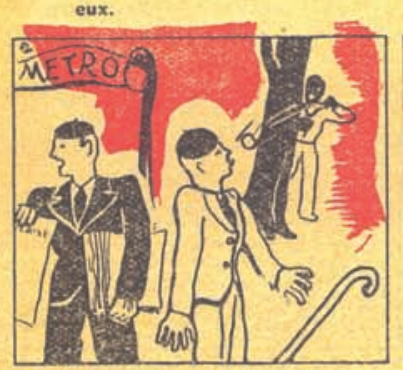

5. Pipe manie habilement la fronde, it le démontre aux

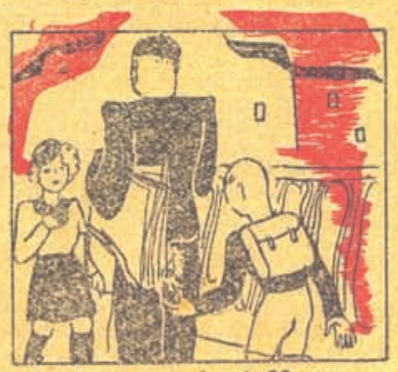

9. Pommes a donné 20 sous. elle attend sa monnaie, Flute travail bion.
X. DU TRAVAIL ANTIFASCISTE

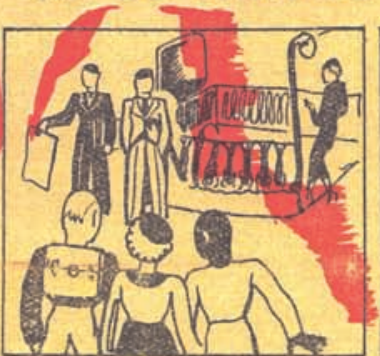

2. Chemin faisant, ils reneontrent 2 fascistes qui vendent leurs lournaux, I'un vend, l'autre le protège muni d'une matraque.

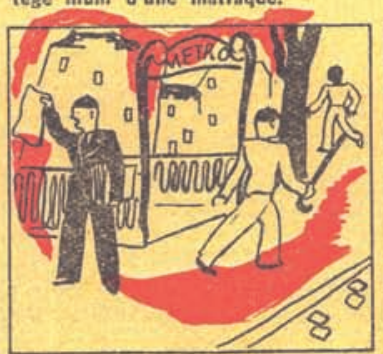

6. ... qui en colère, matraque haute se met a poursuivre le petit nègre.

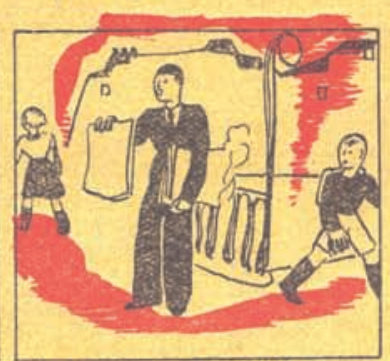

10. Esquivons nous rapidement.

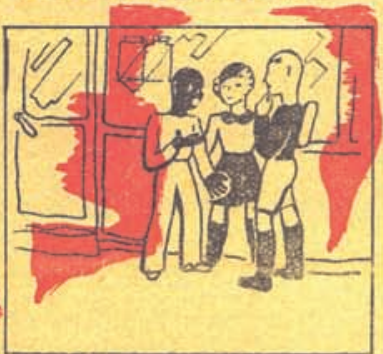

3. II ne faut pas les laisser vendre leur presse, Flute, Pipo moyen de les faire déguerpir.

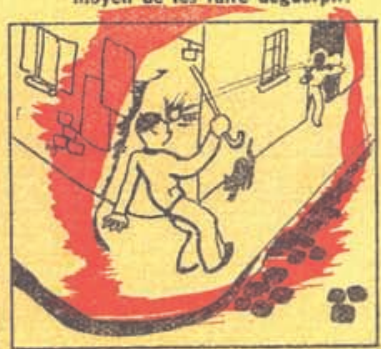

7. Mais Pipe sait maintenir son adversaire a distance.

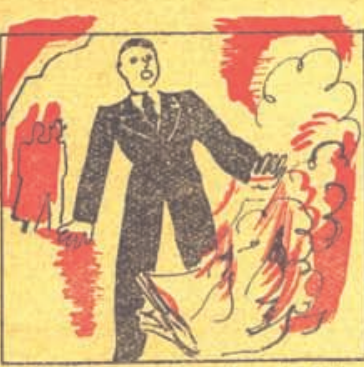

11. pour contempler do loin Tahurissoment des fasciste devant lour journaux en

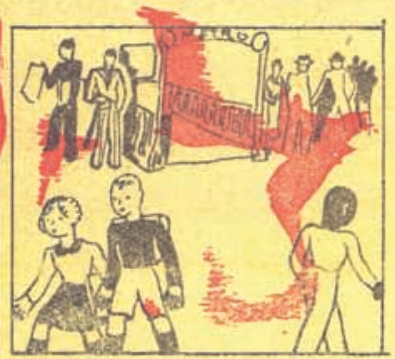

4. Ca y est, lo plan est conçu. A Taction.

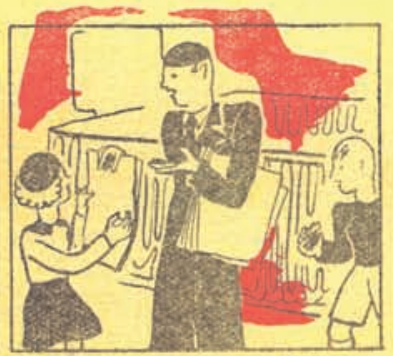

8. Pendant ce temps. Pomma approche le seconde fasciste pour derricere allume son briquet.

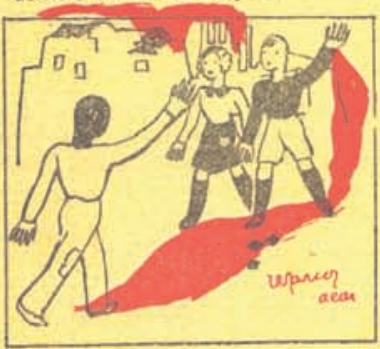

12. Victoire, allons raconter ç? aux copainr.

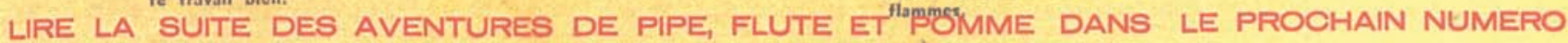




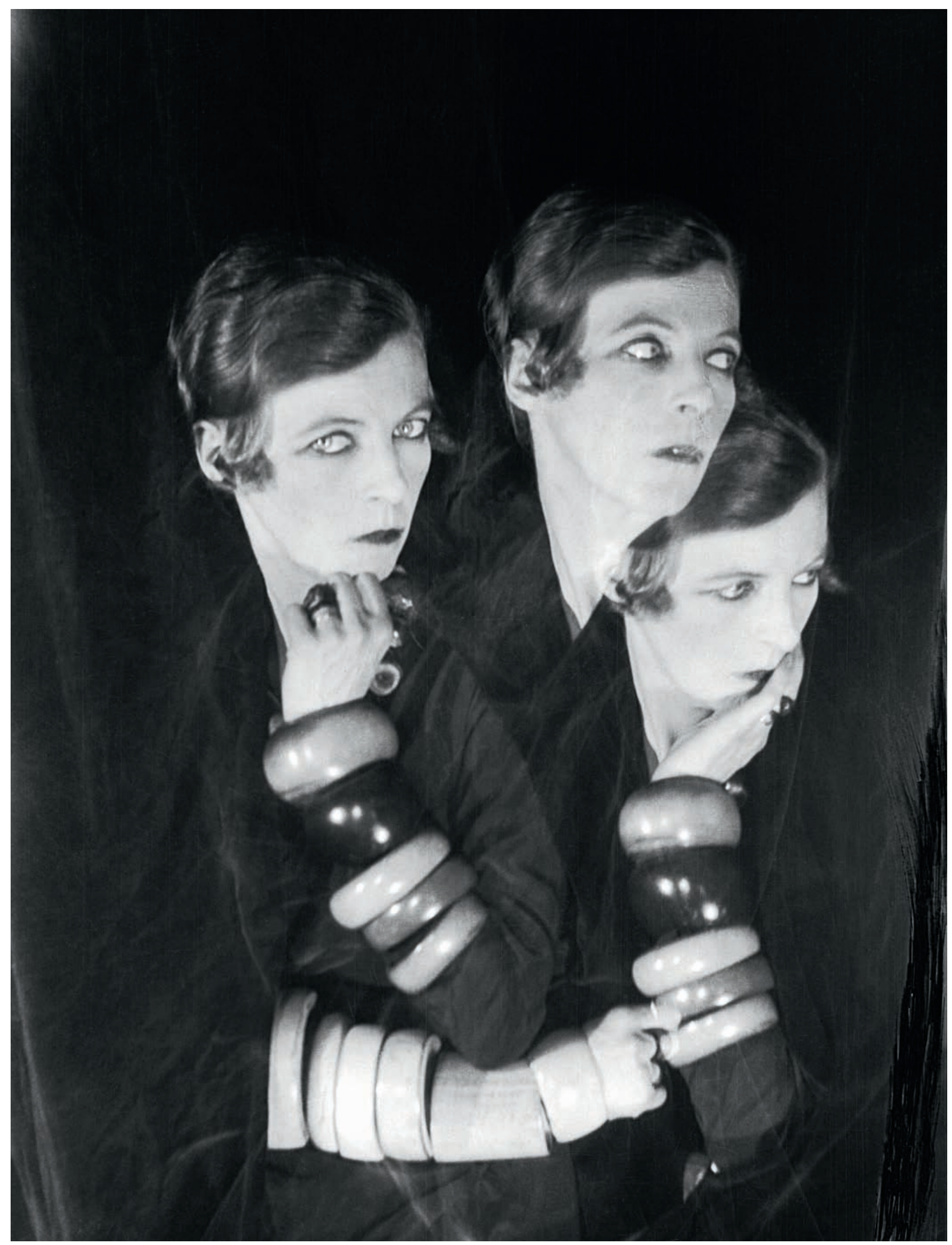


plusieurs contributions (traduites par Beckett ${ }^{38}$ ) de membres du groupe et notamment de son ami Crevel (ibid.: 142), l'auteur de «The Negress in the bordel», figure récurrente dans ses écrits (ibid.: 145), et du tract collectif surréaliste «Murderous humanitarianism ${ }^{39}$ ». Benjamin Péret quant à lui propose un texte sur l'histoire des Noirs au Brésil où il vécut de 1928 à 1931. On peut d'ailleurs supposer que c'est par son intermédiaire que Cunard sélectionne un extrait d'Ensaio Sobre a Música Brasileira (1928), ouvrage pionnier sur la musique brésilienne de Mário de Andrade, intellectuel majeur de son pays. Sadoul, qui avait travaillé avec elle aux éditions Hours Press, analyse dans Negro la représentation du Noir et les clichés racistes véhiculés dans la presse et la littérature enfantine $\mathbf{4 0}^{\mathbf{4}}$. Léon Pierre-Quint, éditeur du premier Manifeste du surréalisme (Paris, Éditions du Sagittaire, 1924), dénonce avec virulence la violence coloniale dans «Races and nations». Enfin, Michelet, proche des surréalistes, est l'auteur de plusieurs contributions importantes. II propose un long texte sur l'histoire de l'Afrique, «African empires and civilisation», ainsi qu'un article au titre éloquent «The white man is killing Africa», très documenté sur les conséquences économiques, politiques et sociales de la colonisation européenne, en Afrique et à Madagascar. La plupart des contributeurs surréalistes de l'anthologie adhèrent à l'Association des écrivains et des artistes révolutionnaires (AEAR), proche du parti communiste, dès sa création en 1932. D'autres contributeurs sont aussi membres de I'AEAR: I'artiste Francis Jourdain, auteur d'un texte sur l'impérialisme français à Madagascar, et, pour la section internationale, Michael Gold, rédacteur en chef de la revue communiste américaine New Masses, et Théodore Dreiser, écrivain américain.

Le racisme de la société dont Cunard est issue et la condition des Noirs américains l'atteignent et l'interpellent surtout à partir de 1928, lorsqu'elle rencontre le pianiste noir américain Crowder. Elle constate alors les violentes réactions que leur histoire sentimentale suscite, notamment auprès de sa mère, Lady Cunard, figure de la haute société londonienne (Fielding 1968). Elle riposte violemment en publiant à compte d'auteur une petite brochure diffusée à son entourage en décembre 1931. La première partie de Black Man and White Ladyship, an Anniversary, attaque ouvertement le racisme de sa mère à l'égard des Noirs tandis que la seconde préfigure Negro Anthology. Elle y dénonce l'esclavage, la discrimination raciale aux États-Unis et les clichés racistes sur l'Afrique. Michelet écrit: «La publication de ce pamphlet marque la rupture bientôt définitive de Nancy avec ses origines, et sera le début d'une nouvelle évolution qui la verra désormais, souvent avec véhémence, aux côtés de ceux que ne satisfait pas un ordre en apparence bien établi. » (Ford 1968: 127) Quelques mois auparavant, Cunard avait publié, dans la revue noire américaine The Crisis ${ }^{\mathbf{4 1}}$, un texte intitulé «Does anyone know any Negroes? ", dans lequel elle faisait déjà le récit des réactions racistes de sa mère vis-à-vis de Crowder.

Crowder lui permet aussi de découvrir une autre Amérique. Dans son livre, As Wonderful As All That?, il s'étonne de l'ignorance de Cunard au sujet de la violence raciale aux États-Unis:

Un soir, lors d'une de nos conversations habituelles d'après le dîner, nous avons abordé la question de la condition des Noirs en Amérique
38. Beckett est le traducteur principal des textes français en anglais. Voir Friedman 2000.

39. Les signataires en sont: André Breton, Roger Caillois, René Char, René Crevel, Paul Éluard, J.M. Monnerot, Benjamin Péret, Yves Tanguy, André Thirion, Pierre Unik et Pierre Yoyotte. Les deux signataires antillais, Monnerot et Yoyotte participent à l'éphémère revue marxiste et surréaliste Légitime Défense publiée par des étudiants martiniquais en 1932 (et censurée). Pour Sophie Leclercq, ce tract, que Crevel rédige en 1932, est une synthèse des positions anticolonialistes du groupe dans l'entre-deuxguerres (2010: 140).

40. À partir de 1933, Sadoul dirige le journal pour enfant Mon camarade, dont les numéros parus après 1935 ont été numérisés par la Bibliothèque nationale de France. En 1938, il écrit un petit pamphlet, "Ce que lisent vos enfants", dans lequel il dénonce la littérature enfantine américaine et fasciste italienne. Je remercie ici Valérie Vigneaux qui m'a permis de faire le lien entre l'article de Sadoul dans Negro et ses activités de rédacteur en chef de Mon camarade.

41. The Crisis, fondé en 1910 par W.E.B Du Bois, est un organe de la National Association for the Advancement of Colored People (NAACP).

\section{ci-contre}

fig. 5

Cecil Beaton, Portrait de Nancy Cunard, vers 1929. Collection National Portrait Gallery NPGx40077.

Courtesy of the Cecil Beaton Studio Archive at Sotheby's. 
42. Bibliothèque du Laboratoire d'anthropologie sociale, archives Michel Leiris, lettre de Nancy Cunard du 3 août 1955 (fr/cdf/las/ FML 01-02-044).

43. Dans la circulaire de 1931, l'ouvrage devait s'appeler Color mais on constate grâce à une lettre de William Carlos Williams adressée à Louis Zukofsky en août 1931 (citée dans Barnett 2007) que, quelques mois après, elle décide de l'appeler Negro, certainement parce qu'il s'agit du terme le plus usité par les Noirs eux-mêmes dans les années 1920-1930. Alain Locke intitule son anthologie New Negro en 1925, les communistes créent en 1928 le journal The Negro Worker et Marcus Garvey édite depuis 1918 The Negro World organe de son association Universal Negro Improvement Association, fondée en 1917.

\section{ci-contre}

fig. 6

Photographie anonyme, Bracelets et main de Nancy Cunard, vers 1950. Harry Ransom Center. The University of Texas, Austin. Archives Nancy Cunar: 2.42 .
[...]. J'étais étonné de l'ignorance absolue de Nancy à ce sujet. [...] elle était très intéressée et désireuse d'apprendre. Je lui parlais des écrivains noirs et lui expliquais où elle pouvait se procurer des livres d'eux et sur eux. Peu à peu, elle a commencé à se construire une bibliothèque sur le sujet. Elle s'abonna à The Crisis, magazine publié par des Noirs à New York. Des journaux noirs américains firent aussi leur apparition. (Crowder et Speck 1987 : 83, notre traduction)

En lui dédiant son livre, Cunard confirme son rôle majeur dans le projet et la réalisation de Negro. L'année de la mort de Crowder, en 1955, elle écrit à Michel Leiris: "J'en suis si triste, hélas, puis c'est entièrement grâce à Henry [...], que "tout" a commencé; je ne l'oublie jamais ${ }^{\mathbf{4 2}}$."

\section{Negro Anthology: une publication partisane aux multiples discours}

En avril 1931, elle adresse une circulaire aux auteurs pressentis pour participer à un ouvrage «entièrement documentaire» consacré à l'histoire des Noirs d'Amérique du Nord et du Sud, d'Europe et d'Afrique ${ }^{43}$.

À l'origine, le plan de ce livre est encore vague. Cunard multiplie les lettres, qu'elle expédie, un peu au hasard, aux amis, à tous ceux qui pourraient, pense-t-elle, lui apporter des documents ou une collaboration quelconque. Le livre se fera ainsi, ballotté de résidence en résidence (car Nancy ne tient pas en place), au gré des réponses et des collaborations qui s'offriront [...]. (Témoignage de Michelet in Ford 1968: 128)

Le contexte de création de Negro, décrit ici par son collaborateur Michelet, explique l'originalité de cet ouvrage mais aussi les contradictions intellectuelles et politiques que tous les auteurs de ce numéro soulèvent, et l'incohérence apparente de son sommaire divisé en sept sections géographiques et thématiques: America, Negro stars, Music, Poetry, West Indies and South America, Europe, Africa.

Negro réunit des textes inédits et compile de nombreux articles, poèmes ou extraits d'ouvrages déjà publiés. On y trouve également un très grand nombre de photographies. L'intérêt de ce corpus iconographique, outre sa taille, réside dans les légendes très précises ou les commentaires personnels de l'éditrice qui accompagnent systématiquement les images. Cette approche souvent subjective participe à la singularité de cette publication non universitaire et totalement artisanale. Enfin, comme Julie Jones le montre dans ce numéro, Negro Anthology s'inscrit aussi dans le courant du documentaire social américain.

Cunard utilise de nombreux documents d'archive bruts, sans commentaires (tracts politiques, extraits de journaux ou d'ouvrages, discours politiques, affiches, témoignages, traités historiques, récits d'esclaves, comptes rendus de jugements...), qui se substituent au texte et créent une prise directe sur le réel. Elle s'appuie ainsi sur des faits concrets et des récits de vies. Ce choix éditorial rend la publication très moderne sur la forme et l'inscrit dans la démarche littéraire des poètes objectivistes américains dont 


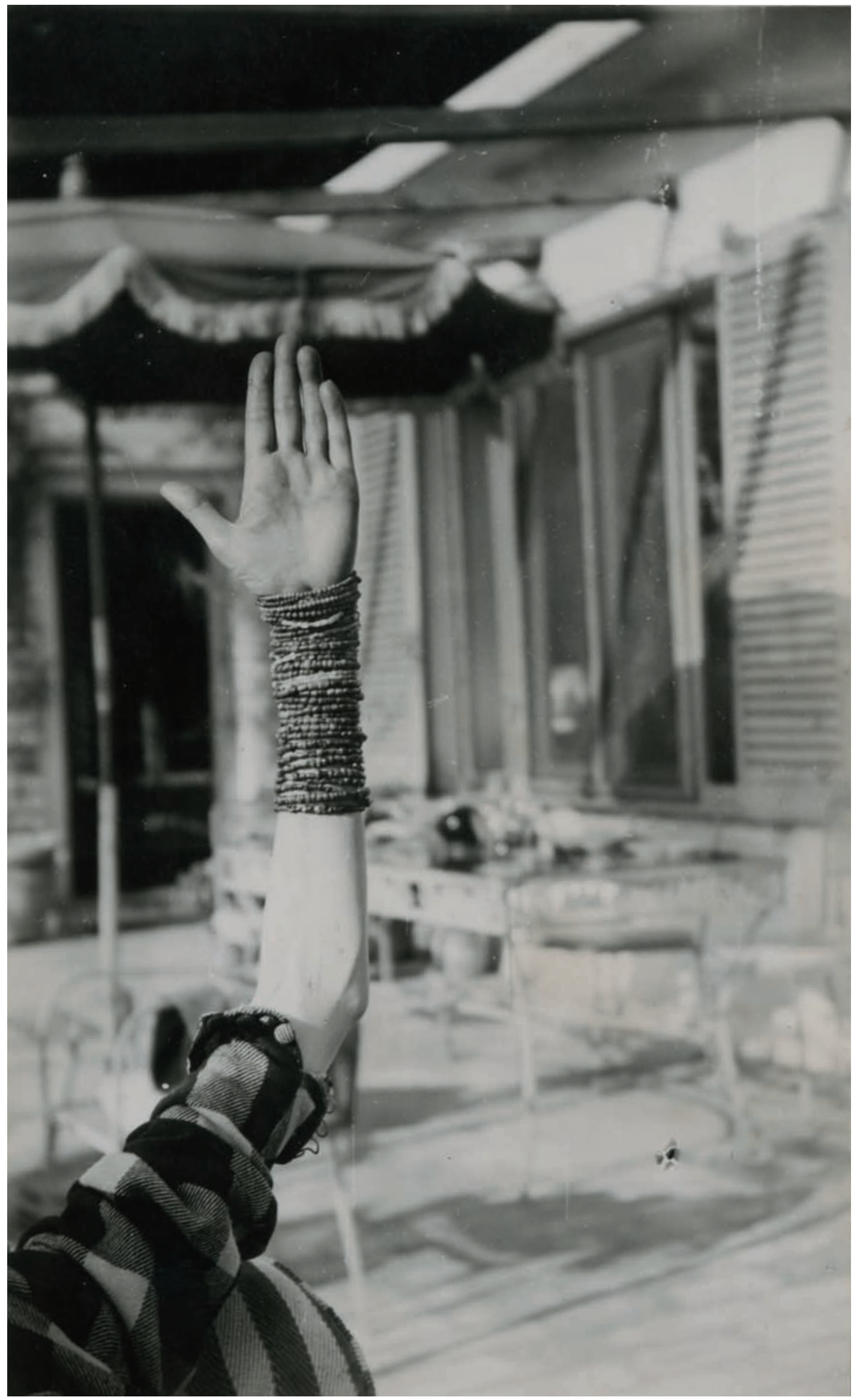




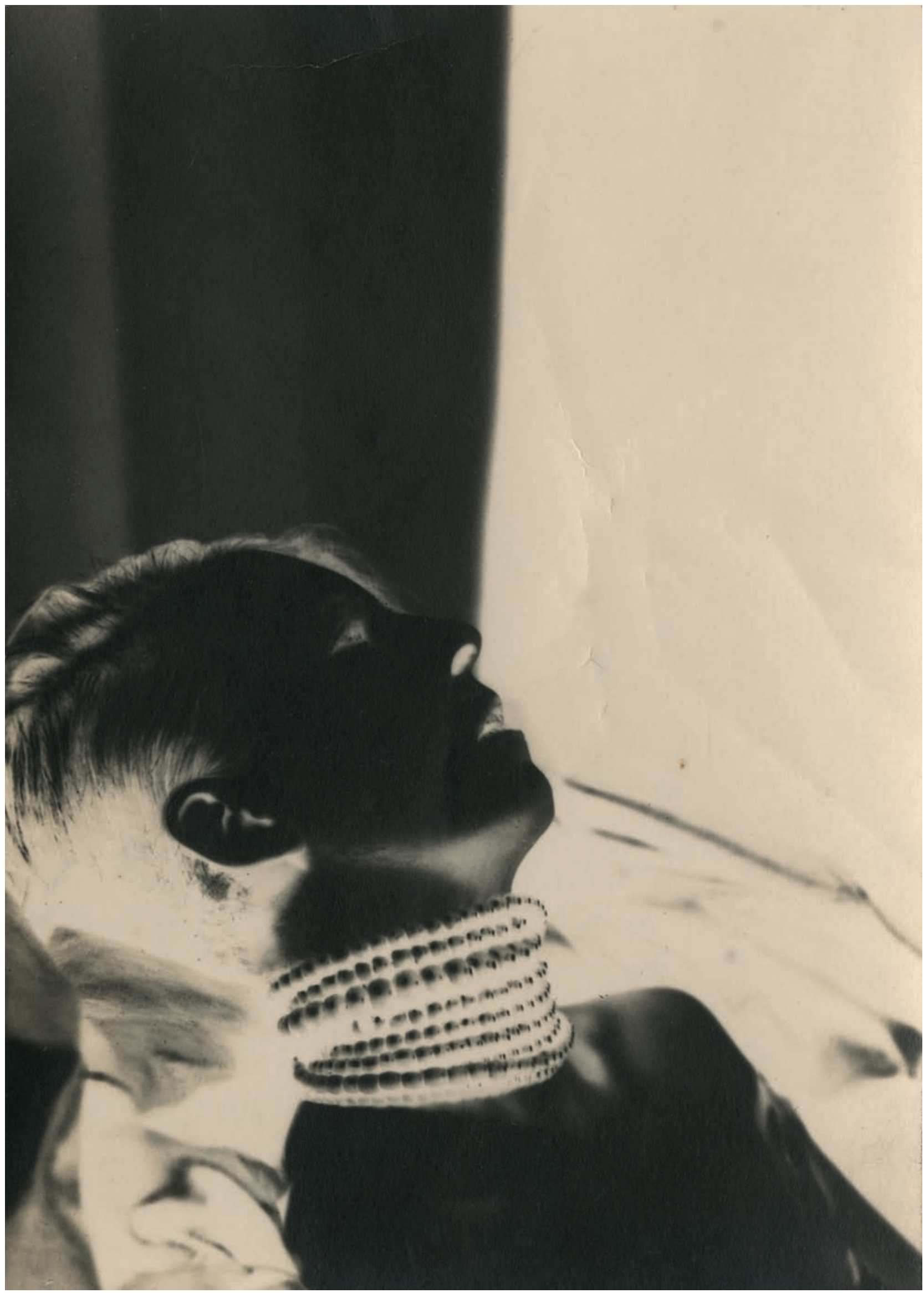


plusieurs sont présents dans l'anthologie (Carl Rakosi, Louis Zukofsky ou encore William Carlos Williams).

Negro Anthology est également une publication engagée et inédite, pour des raisons à la fois politiques et scientifiques. Cunard veut dénoncer la ségrégation raciale, ses conséquences, la violence coloniale et la situation des colonisés. Elle donne pour cela la parole aux militants noirs américains et à ceux des pays colonisés par l'Europe. Leurs textes dénoncent les lynchages, les préjugés raciaux, l'exploitation économique de leurs terres par les Européens et les Américains, le travail forcé, la justice coloniale... D'autres revendiquent la propriété des terres de leurs ancêtres.

L'approche historique, sociale et culturelle de l'Afrique, des Amériques noires et de Madagascar que Cunard propose est aussi une forme d'engagement, bien que certains thèmes ne soient pas novateurs, traités depuis longtemps dans des publications noires américaines ${ }^{\mathbf{4 4}}$. On trouve des textes d'universitaires, de sociologues, d'historiens, d'ethnomusicologues ou d'écrivains qui s'intéressent aux grands empires africains, à la place du Noir dans la littérature européenne, à l'influence des cultures africaines dans les Amériques noires, à l'éducation des Noirs aux États-Unis, à l'acculturation mais aussi aux résistances anciennes et contemporaines (révoltes d'esclaves haïtiens et jamaïcains au xIX siècle, portrait de l'abolitionniste noire américaine Harriet Tubman [1820-1913] ou encore récits de grèves d'ouvriers noirs au Brésil).

La section "Music» est confiée à son ami, le compositeur américain d'avant-garde George Antheil ${ }^{\mathbf{4 5}}$, qui aborde les musiques noires dans leur pluralité formelle et géographique - voir l'article de Yannick Séité dans ce numéro. Pour interroger la place du Noir dans le cinéma, elle fait appel à Kenneth Macpherson qui, en 1929, avait consacré à cette question un numéro spécial de Close Up (la revue d'avant-garde anglophone la plus influente des années 1930 consacrée au cinéma, dont Macpherson était rédacteur en chef) - voir l'article de François Bovier dans ce numéro.

Dans la partie dédiée aux arts plastiques, Cunard sollicite des auteurs européens de référence qui avaient participé dans les années 1920-1930 à la valorisation de l'art africain. Cette section très illustrée rassemble quatre-vingt-dix photographies et douze planches de croquis d'objets issus pour la plupart de prestigieuses collections muséales (British Museum, musée d'Ethnographie du Trocadéro et musée du Congo belge de Tervuren...) et particulières (Paul Guillaume, Félix Fénéon, Charles Ratton, Moffat...). En intégrant les articles consacrés à l'art plastique dans la sous-partie "Art et ethnologie», Cunard s'inscrit dans la lignée des réformateurs du musée d'Ethnographie du Trocadéro ${ }^{46}$ (Leiris, Georges Henri Rivière $\mathbf{4 7}^{\mathbf{4} . .)}$, proches des avant-gardes, qui défendaient alors une approche à la fois esthétique et scientifique des objets, sans toujours se démarquer du contexte colonial ${ }^{48}$. Cette partie, qui constitue pourtant un des inventaires illustrés les plus importants des années 1930, est finalement assez ambiguë, sachant que Cunard avait pour ambition de dénoncer la situation coloniale $\mathbf{4 9}$ et de donner principalement la parole aux Noirs. II est en effet surprenant de ne pas y voir apparaître une contribution du célèbre intellectuel
44. Voir par exemple les sommaires de la revue The Crisis mais aussi la liste des publications de l'Association for the Study of Negro Life and History qui existait depuis 1916 à Washington (ces documents sont consultables à la médiathèque du musée du quai Branly). En 1925 la bibliothèque publique de New York ouvre dans son antenne de Harlem une section dédiée à l'histoire et la littérature des Noirs qui sera particulièrement enrichie en 1926 par l'acquisition, auprès d'Arthur Shomburg (auteur de deux articles dans l'anthologie), de 5000 livres, 3000 manuscrits, 2000 gravures et peintures et plusieurs milliers de brochures, tous consacrés aux Noirs.

45. En 1924, il compose la musique du film expérimental Ballet mécanique de Fernand Léger et Dudley Murphy. Pound lui consacre un livre, Antheil and the Treatise on Harmony. Le 25 mai 1930, il crée Transatlantic à l'opéra de Francfort, représentation à laquelle Cunard et Crowder assistent.

46. À ce sujet, voir les travaux de Jean Jamin, Christine Laurière et Benoît de l'Estoile. En 1932, Cunard sollicite Georges Henri Rivière pour contribuer à l'anthologie mais celui-ci ne lui répond pas (archives du musée de l'Homme: 2 AM 1 K29). Par ailleurs, les archives de l'exposition consacrée au royaume du Bénin, organisée au musée d'Ethnographie du Trocadéro en 1932 par Charles Ratton, révèlent que Cunard était dans la liste des potentiels prêteurs (archives du musée de l'Homme: 2 AM 1 C1b).
47. Tous deux participent à la revue Document (voir à ce sujet les travaux de Denis Hollier et le catalogue de l'exposition The Undercover Surrealism présentée à Londres à la Hayward Gallery en 2006).

48. À ce sujet, voi évidemment les publications de James Clifford et Benoît de l'Estoile, et plus récemment le programme du séminaire "L'ethnologue et le fait colonial » organisé à l'École des hautes études en sciences sociales par Christine Laurière Daniel Fabre et André Mary.

49. Dans son article "Visualizing Africa in Nancy Cunard's Negro Anthology (1934)", M.G. Shanahan analyse de manière très pertinente cette ambiguïté sans pour autant remettre en question l'aspect politique de cet ouvrage (Shanahan 2005). II rappelle notamment que les quatre auteurs de textes sur l'art africain, Ratton, Carl Kjersmeier, Henri Lavachery et Ladislas Szecsi, étaient liés d'une manière ou d'une autre à l'impérialisme européen.

\section{ci-contre}

fig. 7

Barbara Ker-Seymer, Nancy Cunard, 1930. Harry Ransom Center. The University of Texas, Austin. Archives Nancy Cunard: 957:0038:031 (c) The Estate of Barbara Ker-Seymer 
50. À propos de Locke, intellectuel majeur de l'histoire intellectuelle américaine du $x x^{e}$ siècle, voir en français les publications d'Anthony Mangeon.

51. Dans «Miroirs des littératures nègres: d'une anthologie l'autre, revues " (Gradhiva 10, 2009), Anthony Mangeon propose une liste détaillée des auteurs qui publient dans les deux anthologies. On retrouve notamment le professeur John Matheus, I'historien Shomburg, l'anthropologue Melville Herskovitz et le sociologue Edward Franklin Frazier. II est intéressant de signaler que ces deux derniers auteurs s'opposeront sur l'héritage africain dans la culture noire américaine.

52. Sur Zora Neale Hurston, voir l'article d'Emmanuel Parent dans ce numéro.

53. Ce poème est publié pour la première fois en 1926 dans le recueil The Weary Blues (chez Alfred A. Knopf). II est aussi traduit en français en 1931 dans La Revue du monde noir de l'Antillaise Paulette Nardal.

54. Poème également publié en décembre 1932 dans le journal communiste The Negro Worker.

55. Dans une lettre du 28 septembre 1931 (archives Nancy Cunard). Il lui parle notamment de la jeune écrivain Pauly Murray, qui deviendra par la suite une importante militante pour la défense des droits civiques. En 1940, elle précède Rosa Park en refusant de s'asseoir à l'arrière d'un bus, ce qui lui vaut évidemment d'être arrêtée.

56. Langston Hughes avait déjà traduit ces mêmes poèmes pour la revue The Crisis en décembre 1931 Concernant les rapports entre Hughes, Jacques Roumain, Cunard et Nicolás Guillén, voir dans ce numéro le texte de Léon-François Hoffman.
57. On trouve

dans l'anthologie des photographies du frère de Claude McKay. Ce dernier dans la lettre d'août 1932 traduite dans ce numéro par Anthony Mangeon, remercie Cunard pour l'envoi de journaux jamaïcains. A propos de la nonparticipation de McKay, voir dans ce numéro l'introduction d'Anthony Mangeon à la traduction de la lettre.

58. James Ford est le fondateur du journal The Negro Worker, organe de l'association communiste The International Trade Union Committee of Negro Workers fondée en 1928 Cunard connaissait Ford depuis 1931, l'ayant rencontré à Hambourg, où ce dernier vivait, lors de son périple en Allemagne avec Crowder (archives Nancy Cunard, lettre de novembre 1959 adressée à Dorothy Padmore).

59. The Liberator The New Masses, The Daily Worker, The Negro Worker, Le Cri des Nègres..

60. L'expression « ceinture noire" provient de la forme d'un ensemble de treize États majoritairement peuplés de Noirs. À ce sujet, voir l'article d'Amzat Boukari-Yabara dans ce numéro ainsi que la réédition chez Syllepse des textes de C.L.R. James (2012) et de Léon Trotski (2011) sur la question noire aux États-Unis dans les années 1930

61. Fondée en 1910 notamment par Du Bois, pour défendre les droits civiques des Noirs américains.

62. Il est intéressant de signaler que, en 1934 Du Bois quitte la NAACP pour se rapprocher du parti communiste auquel il adhère officiellement en 1961. africain-américain Alain Locke ${ }^{\mathbf{5 0}}$, qui avait déjà écrit sur l'art africain mais aussi organisé à New York une exposition en 1927 (Grossman 2009: 39-44), sans compter qu'il était également l'auteur de l'anthologie The New Negro, parue en 1925. Locke contribue pourtant à l'anthologie de Cunard avec un long texte sur le poète africain-américain Sterling Brown.

Malgré l'unique contribution de Locke, l'héritage du mouvement littéraire et artistique de la Renaissance de Harlem est présent dans l'anthologie de Cunard ${ }^{\mathbf{5 1}}$. Zora Neale Hurston $\mathbf{5 2}^{2}$ et le poète Hughes, symboles notoires du New Negro, tiennent l'une et l'autre une place importante dans Negro. Cunard ouvre son anthologie avec un poème emblématique de Hughes, I Too ${ }^{53}$. Cinq autres poèmes du même auteur apparaissent dans la section poésie, dont le très politique Goodbye Christ ${ }^{\mathbf{5 4}}$. Dans une lettre de septembre 1931, Hughes propose plusieurs auteurs potentiels à Cunard ${ }^{55}$ et lui fait part de ses séjours à Cuba et en Haïti, ce qui laisse supposer que c'est par son intermédiaire que Cunard publie les poèmes de l'Haïtien Jacques Roumain et du Cubain Nicolás Guillén (traduits par lui en anglais $\left.{ }^{56}\right)$. Dans la section consacrée aux Antilles et à l'Amérique du Sud, il est aussi l'auteur d'un essai cinglant sur la situation politique en Haïti, alors sous occupation nord-américaine. Cunard introduit cette même partie avec un long article sur la Jamaïque où elle s'est rendue en 1932, voyage au cours duquel elle a suivi les conseils de l'écrivain jamaïcain Claude McKay, qui ne contribua finalement pas à l'anthologie ${ }^{\mathbf{5 7}}$.

La partie la plus importante de l'ouvrage, «America», reflète une histoire politique plurielle des Noirs de ce pays, qui s'affirme surtout à la fin des années 1920 et au début des années 1930 avec la montée du parti communiste qui s'est emparé de la cause des Noirs Américains au début des années 1920 (voir Shawki 2012). Dans son introduction, Cunard prend clairement position à ce sujet: «L'ordre mondial communiste est la solution au problème noir. » Elle consacre ensuite un chapitre entier à la candidature du Noir américain communiste James Ford ${ }^{58}$ à la vice-présidence des États-Unis. On constate également que les extraits de journaux ou les articles de presse qu'elle retranscrit sont principalement issus de la presse communiste américaine ${ }^{\mathbf{5 9}}$. Mais c'est surtout en soutenant ouvertement le projet de "Black Belt ${ }^{60}$ ", défendu par les communistes américains, que l'anthologie s'inscrit dans les débats de l'époque. En 1928, le sixième Congrès de l'Internationale communiste avait décrété que les Noirs du sud des États-Unis constituaient une nation et qu'il fallait défendre «l'autodétermination pour la Black Belt », dont une carte est reproduite sur la quatrième de couverture de l'anthologie.

En dépit de cet engagement marqué, Negro rassemble de nombreux textes de militants de la National Association for the Advancement of Colored People (NAACP ${ }^{61}$ ) que les auteurs communistes de l'anthologie fustigent violemment, les considérant comme trop bourgeois, modérés et consensuels. Le texte "Blacks and Red» du communiste noir américain Eugene Gordon est particulièrement virulent. II y attaque frontalement deux leaders importants de la NAACP, W.E.B. Du Bois et William Pickens, qui pourtant signent plusieurs textes dans l'anthologie (ibid.). De son côté, Cunard dénonce ouvertement l'association dans son texte au titre significatif, "A reactionary Negro organisation », qui précède la contribution de Du Bois ${ }^{62}$. 
Ces contradictions révèlent en grande partie l'évolution de son engagement politique entre 1931 et 1934. Nous avons vu précédemment que Crowder la sensibilise à la situation des Noirs américains en lui faisant découvrir la revue The Crisis, organe de la NAACP. En 1931, lorsqu'elle part avec lui à New York afin de trouver des contributeurs pour son anthologie, elle rencontre principalement les équipes de The Crisis et des membres éminents de la NAACP. Crowder raconte:

J'introduisis Nancy auprès du personnel de The Crisis [...]. Elle rencontra Walter White, Du Bois, et William Pickens. Pickens nous escorta dans Harlem et présenta Nancy à différentes personnes mais elle ne fut pas toujours bien reçue. Elle était blanche, nous étions noirs, ils ne pouvaient pas oublier cette différence. (Crowder et Speck 1987 : 137-138, notre traduction)

L'année 1931 est aussi celle des «Scottsboro Boys». Le 8 avril, neuf garçons noirs sont jugés de manière expéditive pour le viol de deux Blanches et condamnés à mort ${ }^{63}$. Dès l'annonce du verdict, la NAACP et le parti communiste américain se mobilisent pour faire annuler les condamnations. C'est notamment autour de la manière de défendre les Scottsboro Boys que les deux mouvances s'affrontent. Cunard retranscrit par exemple "Lynching in the quiet manner», article de la militante communiste Josephine Herbst publié en juillet 1931 dans la revue The New Masses, dans lequel celle-ci reproche à la NAACP de ne pas vouloir s'investir officiellement dans la défense des jeunes de Scottsboro. Emblématique du climat raciste du sud des États-Unis, ce procès donne lieu à une mobilisation internationale à laquelle Cunard participe activement ${ }^{64}$. Elle constitue, à Londres, un comité de soutien et prend en 1933 le relais de la campagne internationale lancée par les communistes américains réclamant leur libération. On retrouve évidemment parmi les signataires ses réseaux intellectuels parisiens et londoniens, dont certains auteurs d'Hours Press ${ }^{65}$. C'est dans ce contexte qu'elle se rapproche des communistes, et plus particulièrement du militant trinidadais George Padmore dont elle fait la connaissance en 1932. Amzat Boukari-Yabar montre, dans son article consacré aux militants africains anglophones de l'entre-deux-guerres, l'importance de sa rencontre avec Padmore pour la réalisation de la section consacrée à l'Afrique contemporaine, où l'on retrouve le Kényan Jomo Kenyatta, le Nigérian Ben Nmandi Azikiwe mais aussi le Sud-Africain Edwin Thabo Mofutsanyana, membre fondateur de l'African National Congress (ANC). Par ailleurs, Cunard retranscrit plusieurs articles du Negro Worker ${ }^{66}$ que Padmore dirigeait alors, et dans lequel elle publie en 1933 le poème Lincoln's Grinding Verbiage. Ses nouveaux réseaux se renforcent lors de son second séjour de travail aux États-Unis (printemps 1932) où plus aucun membre de la NAACP ne fait partie de ses interlocuteurs, uniquement recrutés parmi les intellectuels et militants communistes ${ }^{67}$. Sélectionnant ainsi les textes de personnes aux positions désormais éloignées des siennes, de manière non partisane, elle respecte ses objectifs présentés dans l'introduction de Negro: «Inventorier les luttes du peuple noir, les réussites, les persécutions et les résistances qu'elles suscitent. »
63. Voir à ce sujet l'article d'Amzat Boukari-Yabara dans ce numéro, l'ouvrage d'Ahmed Shawki ainsi que les documents réunis en annexe de ce volume.

64. Elle consacre dans Negro un important dossier à ce sujet auquel elle contribue personnellement avec l'article «Scottsboro and other Scottsboro " qui mêle comptes rendus du procès, description de la mobilisation internationale, listes de lynchages...

65. Dans une lettre de janvier 1933, Péret lui raconte qu'il a fait signer plusieurs feuilles de soutien à cet appel à des membres de l'AEAR. II lui donne aussi les coordonnées d'intellectuels à solliciter, au Brésil évidemment, mais aussi en Roumanie ou en Belgique (archives Nancy Cunard). Cette pétition fut signée par de nombreux contributeurs de l'anthologie, Kjersmeier mis à part.

On trouve parmi les signataires Lye, Gertrude Stein, Natalie Barney, Darius Milhaud, Beaton, Key-Seymer, Jacob Epstein, Ossip Zadkine, les Sitwell...).

\footnotetext{
double page suivante

fig. 8

Photographie anonyme,

Bracelets et trompes en ivoire de la collection Nancy Cunard, vers 1930. Harry Ransom Center. The University of Texas, Austin. Archives Nancy Cunard: 957:038: 1044-1046.

fig. 9

Jacques-André Boiffard, Intérieur de la maison de Nancy Cunard à La ChapelleRéanville dans les années 1930. Harry Ransom Center. The University of Texas, Austin. Archives Nancy Cunard: 57-038-916-920.
}

66. À propos de la revue The Negro Worker (1928-1937), voir la présentation de Susan Campbell sur le site http://marxists.anu.edu.au, qui a mis en ligne les sommaires de la revue, mais aussi Dewitte 1985.

67. Elle rencontre les collaborateurs de la publication The New Masses, et notamment Laurence Gellert et John Spivak. Gellert publie dans l'anthologie un long article consacré au Negro song of protest de Caroline et de Géorgie, dans lequel il retranscrit des chants déjà publiés dans la revue The New Masses. Spivak est l'auteur de Georgia Nigger (1932), ouvrage issu d'une enquête menée entre 1930 et 1931 sur les prisons, les camps de travail et les chaînes de forçats (chain gang) en Géorgie. Cunard sélectionne pour son anthologie plusieurs illustrations de son livre, reproduites ici dans l'article de Julie Jones. 


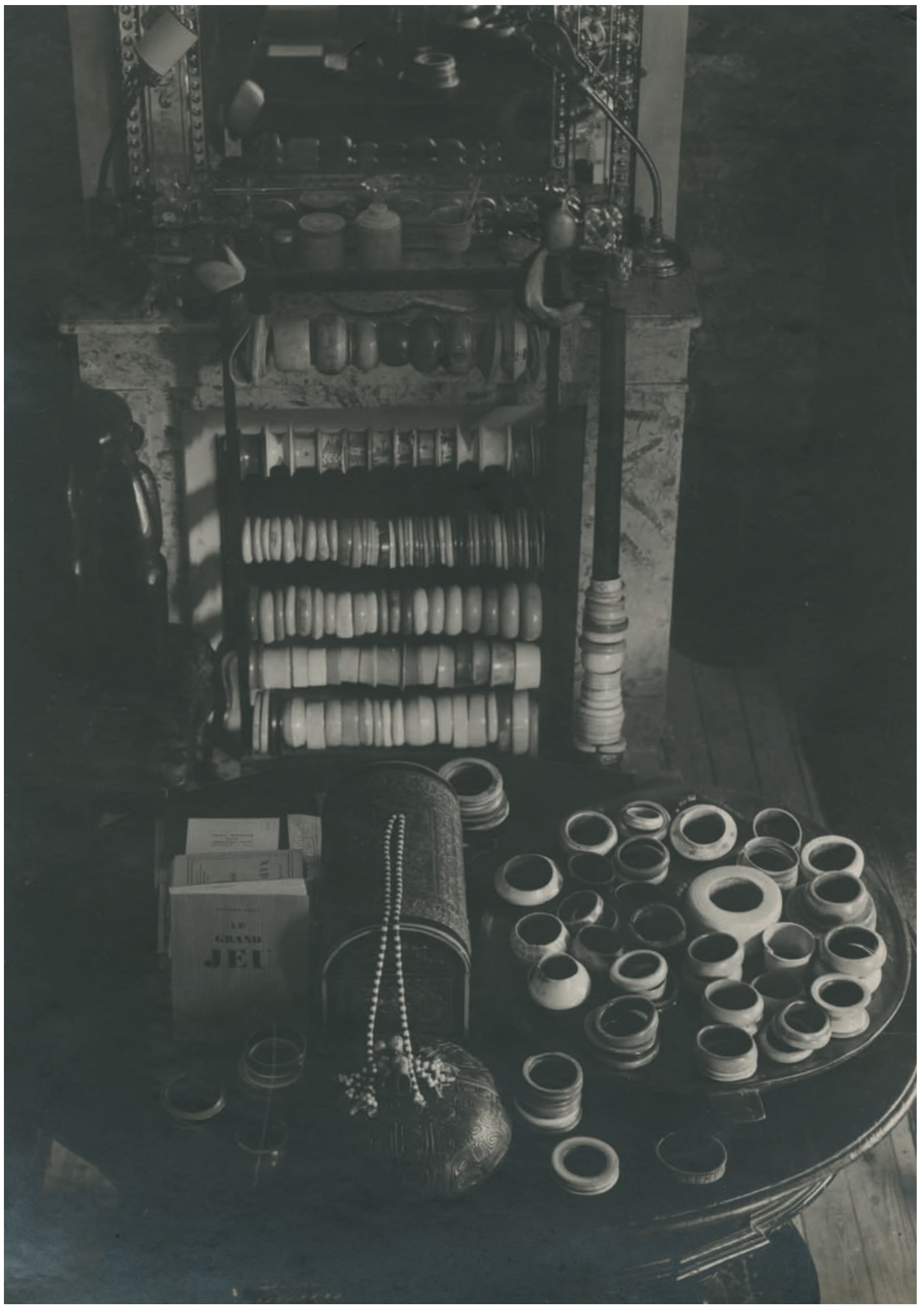




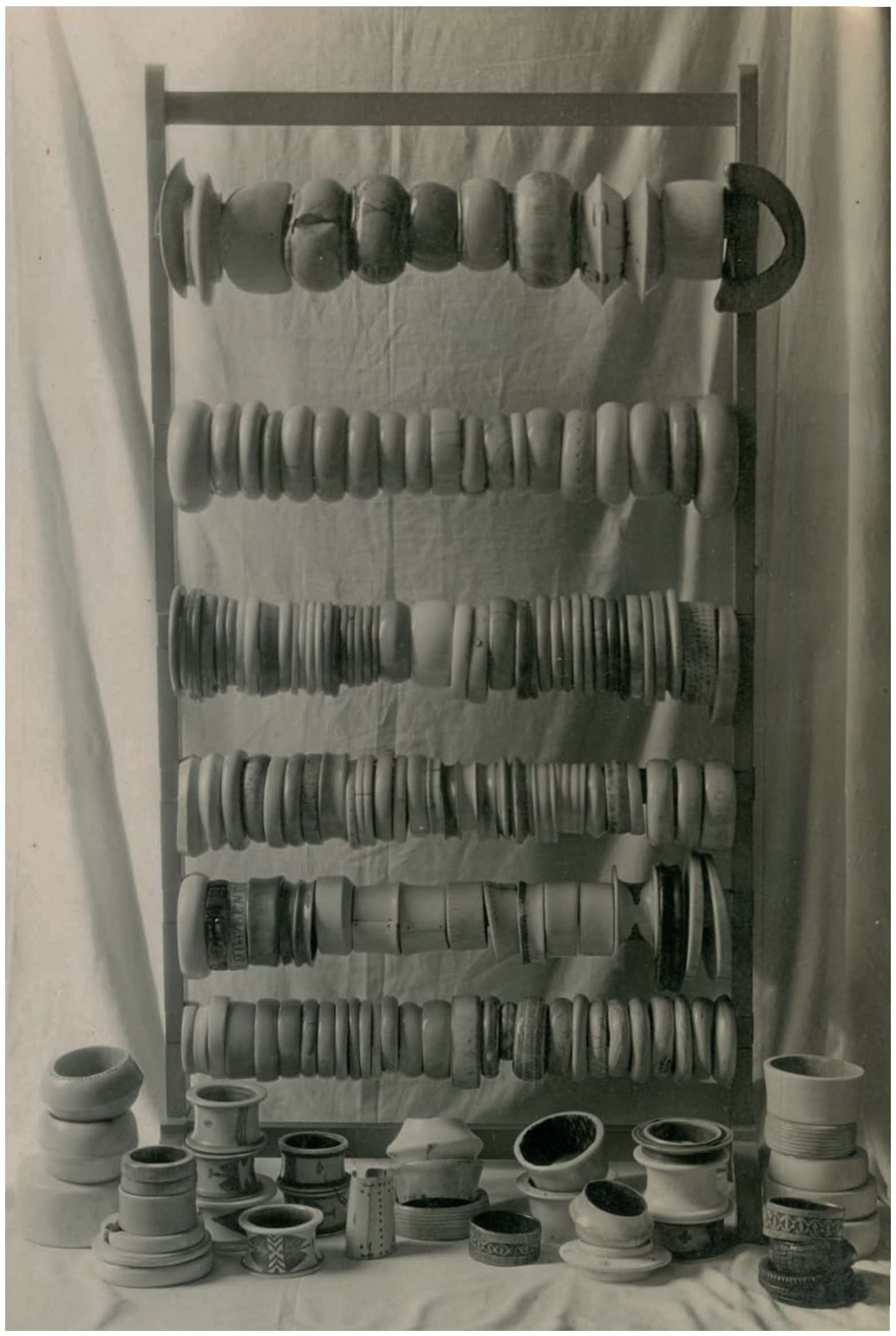




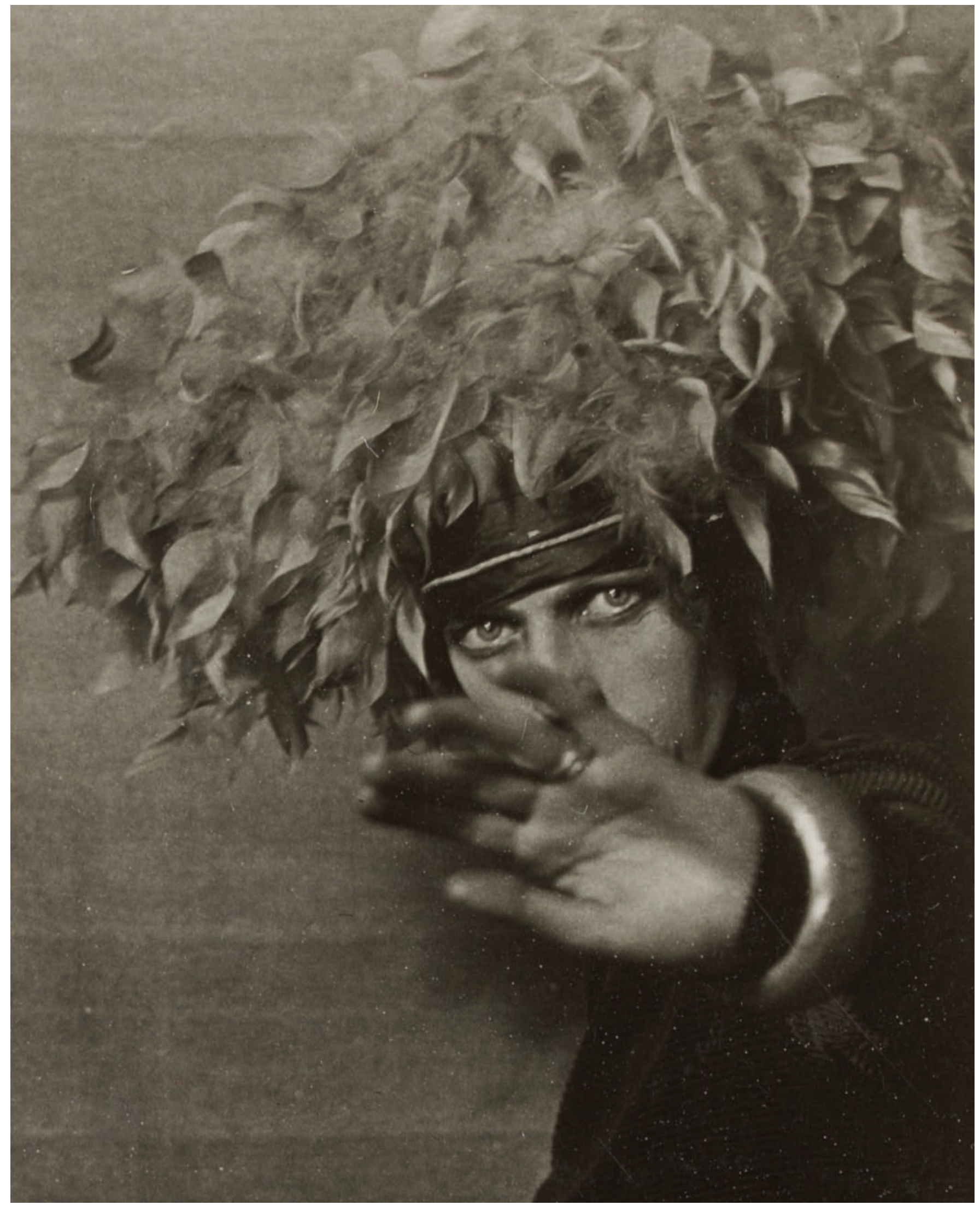


À sa sortie, Negro Anthology bénéficie d'un réel succès d'estime dans les milieux intellectuels, journalistiques et politiques africains-américains ${ }^{\mathbf{6 8}}$, ce dont attestent les nombreuses lettres reçues par Cunard. Locke la félicite «pour la meilleure anthologie, dans tous les sens du mot, jamais réalisée à propos des Noirs ${ }^{69}$ ». La militante Mary McLeod Bethune la remercie pour l'intérêt que l'anthologie porte aux femmes noires américaines. Un journaliste du Chicago Defender la félicite et lui suggère d'éditer une version moins coûteuse pour la rendre accessible aux plus pauvres. Mais, malgré les éloges, le livre ne connaît pas le succès attendu. II est censuré dans les Antilles britanniques. Les quelques centaines d'exemplaires que Cunard et son éditeur avaient conservés sont détruits pendant la guerre. L'ouvrage est aujourd'hui une rareté ${ }^{71}$.

Après Negro, Cunard s'investit dans le journalisme et écrit dans la presse de gauche anglo-saxonne, notamment The Left Review et l'Associated Negro Press de Chicago. En 1935, elle couvre les débats de la Société des Nations lors de l'invasion de l'Éthiopie et se mobilise pour soutenir les Éthiopiens (voir l'article d'Amzat Boukari-Yabara dans ce numéro et Cunard 2002). L'autre tournant de sa vie se situe en 1936, lorsqu'elle part en Espagne suivre les événements de la guerre civile et s'engage, comme de nombreux intellectuels, auprès des républicains espagnols. Elle rencontre alors Pablo Neruda, avec qui elle fonde, en 1937, l'éphémère revue Les poètes du monde défendent le peuple espagnol (six numéros), qu'ils impriment dans sa maison de La Chapelle-Réanville, et à laquelle contribuent Hughes, Guillén, W.H. Auden et Tzara, entre autres ${ }^{71}$.

Elle continue aussi à fréquenter les militants africains et antillais et publie en 1942 le pamphlet anticolonialiste The White Man's Duty, rédigé sous forme de dialogue entre elle et Padmore. Trois ans plus tard, elle réédite, sous forme de brochure, l'article de Michelet «African empires and civilization" aux éditions International African Service Bureau Publications, dont les éditeurs principaux sont Padmore, T.R. Makonnen, Kenyatta, Wallace Johnson, C.L.R. James et Peter Abrahams.

Malgré certaines contradictions et certains poncifs primitivistes et essentialistes évoqués par tous les auteurs de ce numéro, Negro Anthology reste une synthèse majeure, unique et originale de la diversité des discours scientifiques, politiques et culturels des Noirs et sur les Noirs dans les années 1930. «On connaît en France Nancy Cunard. Elle y a passé la majeure partie de sa vie et plus tard on ne pourra faire, sans parler d'elle, l'histoire intellectuelle d'une part de ce siècle." Lorsque Aragon écrit ces quelques lignes dans Les Lettres françaises en 1960, il pense certainement à l'histoire intellectuelle européenne (blanche) et n'imagine pas que Cunard contribue également à l'histoire du panafricanisme du xxe siècle.
68. Nous n'avons pas trouvé de document concernant la réception de Negro dans les milieux intellectuels européens à l'époque de sa sortie. Les témoignages souvent élogieux et admiratifs des Européens sont compilés dans Ford 1968

69. Archives Nancy Cunard, lettre du 14 avril 1934.

70. En avril 1963, Hughes lui écrit fièrement qu'il a vu Negro dans un catalogue de vente de livres rares (archives Nancy Cunard).

71. La même année, en collaboration avec la revue The Left Review, elle édite la brochure «Authors take sides. On the Spanish War. 148 contributions " dans laquelle elle interpelle les écrivains anglo-saxons sur la guerre d'Espagne en leur demandant de se positionner sur le sujet. Celle-ci est introduite par un texte de soutien aux républicains espagnols signé par Aragon, W.H. Auden, José Bergamin, Jean-Richard Bloch, Cunard, Brian Howard, Heinrich Mann, Ivor Montagu, Neruda, Ramon Sender, Stephen Spender et Tzara.

\section{ci-contre}

fig. 10

Curtis Moffat, Nancy Cunard, 1926 (C) Curtis Moffat / Victoria and Albert Museum, London. 


\section{Collectif}

2011 "Artistes engagez-vous ", Aden 10.

\section{Aragon, Louis}

1974 « J'appelle poésie cet envers du temps ", in L'CEuvre poétique, tome IV (1927-1929). Paris, Livre Club Diderot.

2002 La Défense de l'infini, Lionel Follet (éd.). Paris, Gallimard.

2011 Lettres à André Breton 1918-1931, Lionel Follet (éd.). Paris, Gallimard

\section{Arlen, Michael}

2013 Le Chapeau vert. Paris, Les Belles Lettres.

\section{Barnett, Anthony}

2007 Listening for Henry Crowder. A Monograph on His Almost Lost Music. Lewes, Allardyce, Barnett.

\section{Boittin, Jennifer Anne}

2010 Colonial Metropolis. The Urban Grounds of AntiImperialism and Feminism in Interwar Paris. Lincoln, University of Nebraska Press.

\section{Broderick, John C.}

1977 «Paris between the wars: An unpublished memoir by Solita Solano ", The Quarterly Journal of the Library of Congress XXXIV (7).

\section{Buot, François}

2008 Nancy Cunard. Paris, Pauvert.

\section{Chadwick, Withney} et Latimer, Tirza True

2003 The Modern Woman Revisited. Paris Between the Wars. New Brunswick Rutgers University Press.

\section{Chisholm, Anne}

1980 Nancy Cunard.

Paris, Olivier Orban

\section{Chivallon, Christine}

2004 La Diaspora noire des Amériques. Expériences et théories à partir de la Caraibe. Paris, CNRS.

\section{Crowder, Henry et Speck, Hugo}

1987 As Wonderful As All That? Henry Crowder's Memoir of His Affair with Nancy Cunard, 1928-1935.

Navarro, Wild Trees Press.

\section{Cunard, Nancy}

1969 These Were The Hours. Memories of My Hours Press, Reanville and Paris, 1928-1931. Hugh Ford (éd.). Carbondale, Southern Illinois University Press.

2002 Essays on Race and Empire, Maureen Moynagh (éd.) Peterborough, Broadview Press.

\section{Cunard, Nancy (éd.)}

1934 Negro Anthology. Londres, Wishart \& Co (rééditions abrégées: Ford, Hugh [éd.], Negro: An Anthology, Frédérick Ungar Publishing Co., 1970; New York-Londres,

Continuum, 1996/2002)

\section{Dewitte, Philippe}

1985 Les Mouvements nègres en France 1919-1939. Paris, L'Harmattan.

\section{Edwards, Brent Hayes}

2003 The Practice of Diaspora. Literature, Translation and The Rise of Black Internationalism. Cambridge, Harvard University Press.

\section{Fabre, Michel}

1985 La Rive noire. De Harlem à la Seine. Paris, Lieu Commun.

\section{Fielding, Daphne}

1968 Emerald and Nancy. Lady Cunard and her Daugther. Londres, Eyre and Spottiswoode.

\section{Ford, Hugh}

1968 Nancy Cunard: Brave Poet, Indomitable Rebel 1896-1965. New York, Chilton Book Company.

1996 Published in Paris.

L'édition américaine et anglaise à Paris, 1920-1939.

Paris, IMEC éditions.

\section{Friedman, Alan Warren}

2000 Beckett in Black and Red. The Translations for Nancy Cunard's Negro (1934). Lexington,

The University Press of Kentucky.

\section{Gilroy, Paul}

2003 L'Atlantique noir. Modernité et double conscience. Paris, Kargo.

\section{Grossman, Wendy}

2009 Man Ray, African art, and the Modernist Lens. Washington -Minneapolis, International Arts \& Artists et The University of Minnesota Press.

\section{Latimer, Tirza True}

2005 «Visions émancipatrices. Portraiture et identité sexuelle dans le Paris des années 20 ", Clio. Histoire, femmes et sociétés, $n^{\circ} 22$.

\section{Leclercq, Sophie}

2010 La Rançon du colonialisme. Les surréalistes face aux mythes de la France coloniale (1919-1962). Dijon, Les Presses du réel.

\section{Mangeon, Anthony}

2009 « Miroirs des littératures nègres: d'une anthologie l'autre, revues", Gradhiva 10 ["Présence Africaine. Les conditions noires: une généalogie des discours "].

\section{Marcus, Jane}

2000 Hearts of Darkness. White Women Write Race. New Brunswick, Rutgers University Press.

\section{Moeglin-Delcroix, Anne}

1997 Esthétique du livre d'artiste (1960-1980). Paris, Jean-Michel Place et Bibliothèque nationale de France.

\section{Saïd, Edward}

2000 Culture et Impérialisme. Paris, Fayard.

\section{Séité, Yannick}

2010 Le Jazz à la lettre.

Paris, PUF.

\section{Shanahan, M.G.}

2005 «Visualizing Africa

in Nancy Cunard's Negro Anthology (1934) », Journal of Colonialism and Colonial History.

\section{Shawki, Ahmed}

2012 Blacks and Red Les Mouvements noirs et la gauche américaine 1850-2010. Paris, Éditions Syllepse. 


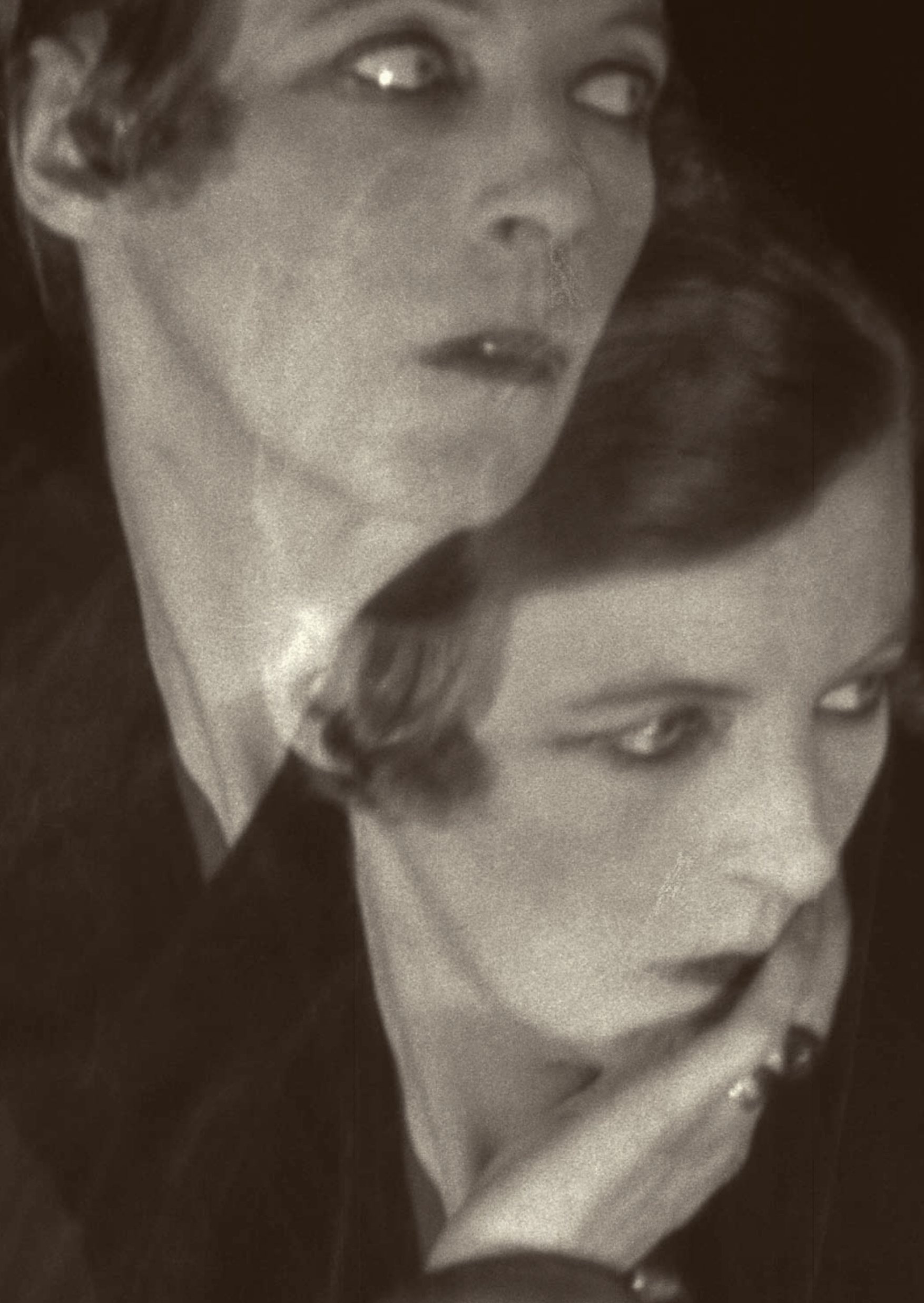

